\title{
Regulation of transcriptional activators by DNA-binding domain ubiquitination
}

\author{
Vivien Landré ${ }^{1}$, Bhindu Revi ${ }^{1}$, Maria Gil Mir ${ }^{1}$, Chandra Verma ${ }^{2,3,4}$, Ted R Hupp${ }^{1}$, Nick Gilbert ${ }^{5}$ and Kathryn L Ball ${ }^{\star, 1}$
}

Ubiquitin is a key component of the regulatory network that maintains gene expression in eukaryotes, yet the molecular mechanism(s) by which non-degradative ubiquitination modulates transcriptional activator (TA) function is unknown. Here endogenous p53, a stress-activated transcription factor required to maintain health, is stably monoubiquitinated, following pathway activation by IR or Nutlin-3 and localized to the nucleus where it becomes tightly associated with chromatin. Comparative structure-function analysis and in silico modelling demonstrate a direct role for DNA-binding domain (DBD) monoubiquitination in TA activation. When attached to the DBD of either p53, or a second TA IRF-1, ubiquitin is orientated towards, and makes contact with, the DNA. The contact is made between a predominantly cationic surface on ubiquitin and the anionic DNA. Our data demonstrate an unexpected role for ubiquitin in the mechanism of TA-activity enhancement and provides insight into a new level of transcriptional regulation.

Cell Death and Differentiation (2017) 24, 903-916; doi:10.1038/cdd.2017.42; published online 31 March 2017

Controlling the rate at which individual genes are expressed is fundamental to development, homeostasis and healthy ageing in eukaryotes. Underpinning the core transcriptional machinery is a complex network of transcriptional activators (TAs) that ensure tight regulation of gene expression. ${ }^{1,2}$ It has become clear that ubiquitin and the proteasome have key roles in the regulatory network that maintains transcriptional control. ${ }^{3-5}$ Yet, although there is a strong link between the rate at which TAs are degraded and their ability to drive expression from target promoters, the molecular mechanisms by which ubiquitin can activate transcription are poorly understood.

The role of the UPS in the regulation of p53, a wellcharacterized transcription factor and tumour-suppressor protein, has been studied extensively. ${ }^{6-8}$ However, there is a paucity of studies linking site-specific ubiquitination to physiological outcome. In part, this is because p53 displays a complex pattern of potential ubiquitin-acceptor sites. ${ }^{9,10}$ Although ubiquitination has generally been linked to the negative regulation of $\mathrm{p} 53$, studies on the activated endogenous p53-pathway ${ }^{11,12}$ and on E4F1-mediated modification of $p 53^{13}$ suggest that ubiquitination may be involved in upregulating this TA under some cellular conditions. MDM2 is the major, and best-characterized, E3-ligase for p53. Under some conditions, MDM2 can catalyse polyubiquination, as well as, p53 monoubiquitination ${ }^{14}$ and this depends on the recruitment of E4-ligases such as, UBE4B-ligase ${ }^{15}$ or MDM4. ${ }^{16}$

Here the monoubiquitination of p53 is linked to its nuclear localization and chromatin-binding activity. Strikingly, in cells where the p53-pathway has been activated, polyubiquitination is switched to stable p53 monoubiquitination. Using comparative structure/function analysis in combination with in silico modelling and cell-based transcription assays, we demonstrate that modification at DBD Lys residues with monoubiquitin is sufficient to support TA function through a direct effect on DNA-binding activity.

\section{Results}

Nutlin and IR induce stable monoubiquitination of p53. The role of p53 in tumour suppression is closely linked with its sequence-specific DNA-binding activity and transactivation of genes required for growth control and cellular homeostasis. Intense efforts have therefore focused on activating p53 as a potential therapeutic avenue. Nutlin, for example, is a cisimidazoline analogue, which binds to the MDM2 hydrophobic pocket $^{17}$ (Figure 1a) inhibiting its transrepressor function ${ }^{18}$ but not its ability to ubiquitinate p53. ${ }^{12}$ Consistent with Nutlin being an allosteric activator of MDM2 E3-activity, we have previously noted that Nutlin-mediated activation of p53, exemplified here by induction of the downstream targets p2 $1^{\text {WAF1 }}$ and MDM2 (Figure 1b), is accompanied by the production of high molecular weight forms of $p 53 .^{12,18}$ To establish whether these high molecular weight forms of p53 (Figure 1c) represented ubiquitin adducts, the ubiquitinated proteome was isolated from cells expressing His-ubiquitin (Figure 1d). When the His-ubiquitin modified proteins were analysed the profile of endogenous polyubiquitinated p53 present in control cells (Lane 2) was strikingly altered by Nutlin-3 (Lane 3). Specifically, Nutlin-3 significantly enhanced (multi-) monoubiquitination of p53 and there was a loss of polyubiquitination. Thus, in control cells p53 is subject to

\footnotetext{
${ }^{1}$ Cell Signalling Unit, ECRC, IGMM, University of Edinburgh, Edinburgh EH4 2XR, UK; ${ }^{2}$ Bioinformatics Institute (A*STAR), 30 Biopolis Street, 07-01 Matrix Singapore 138671, Singapore; ${ }^{3}$ Department of Biological Sciences, National University of Singapore, 14 Science Drive 4, Singapore 117543, Singapore; ${ }^{4}$ School of Biological Sciences, Nanyang Technological University, 60 Nanyang Drive, Singapore 637551, Singapore and ${ }^{5}$ MRC Human Genetics Unit MRC, IGMM, University of Edinburgh, Edinburgh EH4 2XR, UK

${ }^{*}$ Corresponding author: KL Ball, IGMM Edinburgh Cancer Research Centre, University of Edinburgh, Western General Hospital, Crewe Road South, Edinburgh EH4 2XR, UK. Tel: +44 (0) 131651 8500; Fax: +44 (0) 131651 8800; E-mail: Kathryn.Ball@igmm.ed.ac.uk

Received 23.7.16; revised 14.2.17; accepted 16.2.17; Edited by X Lu; published online 31.3.2017
} 
polyubiquitination, consistent with its short half-life and proteasome-mediated degradation; ${ }^{19}$ whereas in Nutlin-3treated cells, p53 is predominantly monoubiquitinated leading us to speculate that Nutlin-3-mediated ubiquitination may have a role in regulating p53 activity rather than degradation.
Notably, after Nutlin-3 treatment, unmodified p53 was isolated together with the monoubiquitinated protein (Figure 1d, lane 3), this is in line with our observation that monoubiquitinated p53 can form mixed tetramers with unmodified p53 (unpublished data).

b

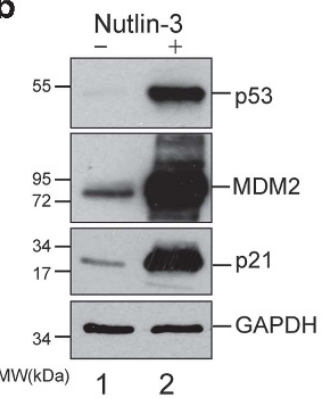

c
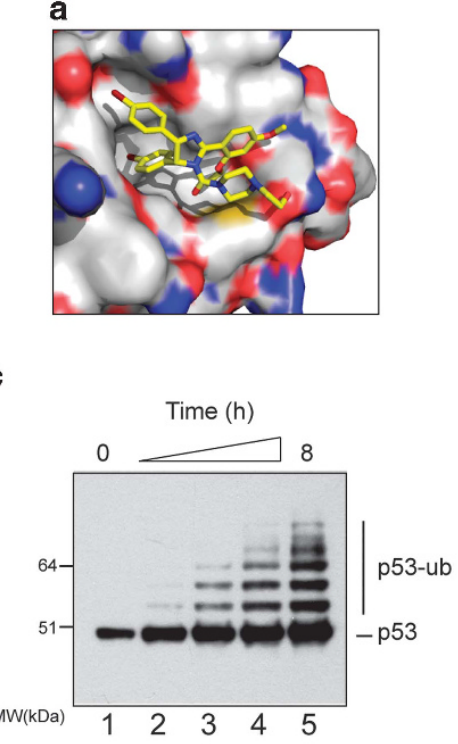

d

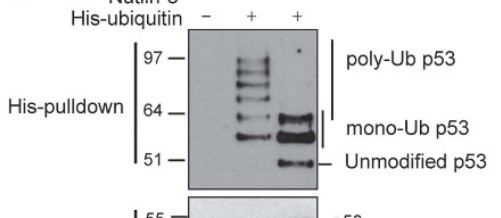

Direct Lysis $\left.\left.\right|_{\text {MW(KDa) }}\right|_{25} ^{55}$

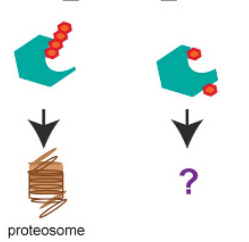

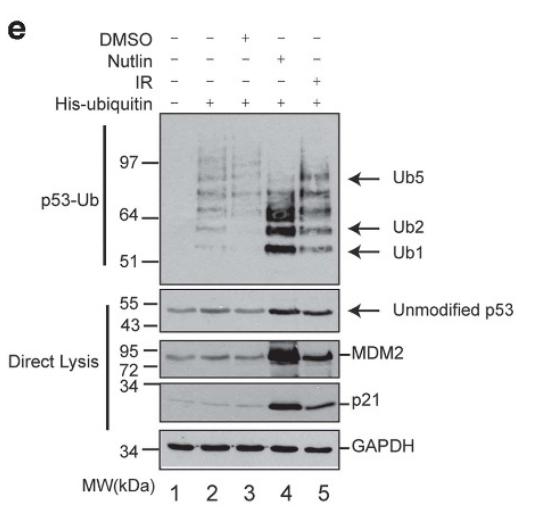

f

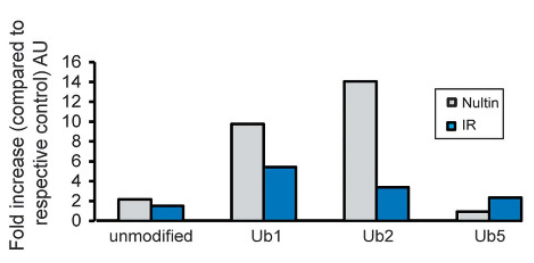

9

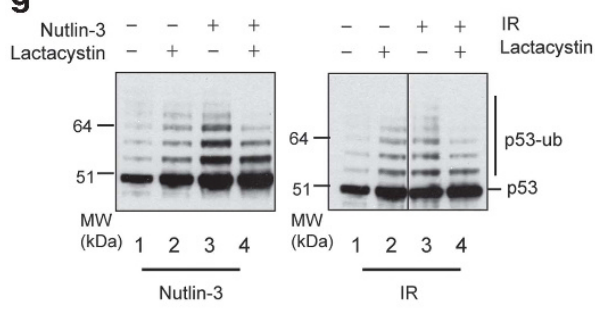

i

Time + cycloheximide ( $\mathrm{min})$

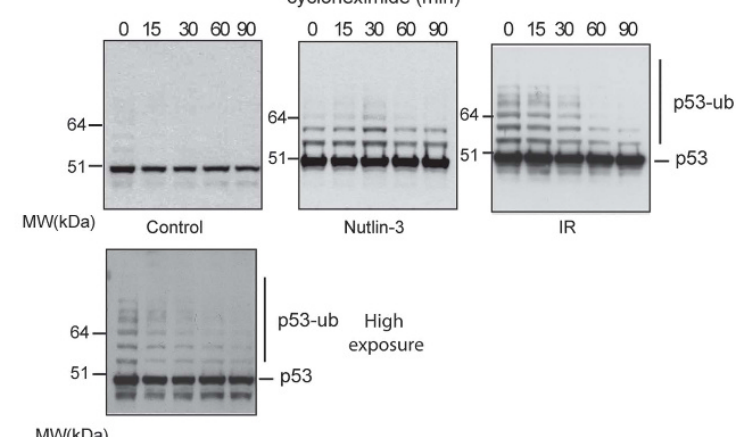

$\mathrm{MW}(\mathrm{kDa})$ 
Based on previous studies ${ }^{11}$ showing exposure of cells to IR increased p53 ubiquitination, we investigated whether IR, like Nutlin-3, led to enhanced monoubiquitination of p53. In agreement with Maki and Howley, ${ }^{11}$ we found that IR produced a substantial change in His-ubiquitinated endogenous p53 (Figure 1e) with an increase in both mono- and polyubiquitination. The mixture of ubiquitin adducts likely reflects the fact that IR has a transient effect on the steady-state levels of $p 53,{ }^{20}$ whereas the Nutlin-3 effect is longer lived. ${ }^{12}$ However, by quantifying the first and second monoubiquitin bands (Figures 1e and f; Ub1 and Ub2) relative to the control samples (Figure 1e) we also found enrichment of monoubiquitinated p53 in IR-treated cells. Thus, although Nutlin-3 produces a greater increase in monoubiquitinated p53, IR also reproducibly favours the generation of p53-monoubiquitin forms (Figure 1f).

It is unclear whether stable monoubiquitination in Nutlin- or IR-treated cells is linked to signalling events in the p53activation pathway. We began to address this issue by determining whether Nutlin-3- and IR-stimulated monoubiquitination was coupled to p53 degradation. p53 protein levels increased and, as expected for a short-lived protein, a portion of the TA accumulated in an ubiquitinated form when the proteasome was inhibited using lactacystin (Figure 1g). However, proteasome inhibition did not lead to the accumulation of Nutlin-3- or IR-induced p53 forms; in fact there was a reproducible decrease in ubiquitinated $p 53$ relative to the unmodified protein in cells treated with either agent plus lactacystin (Figure 1g). This is consistent with findings that monoubiquitin is removed from substrates under conditions of proteotoxic stress. ${ }^{21}$ Further, although the basal half-life of p53 was approximately $20 \mathrm{~min}$ (Figure 1h), in Nutlin-3 or IR-treated cells, the $t_{0.5}$ increased to $>90 \mathrm{~min}$ (Figure 1h). These two experiments indicate that enhanced modification of p53 following pathway activation does not signal degradation. Further, when the turnover of the ubiquitinated p53 forms was monitored in control cells, they had a short half-life of $<15$ min (Figure 1i; Ub-p53) consistent with these being rapidly degraded K48-polyubiquitinated p53-intermediates. Strikingly, the mono- and multi-monoubiquitinated forms of p53 detected in the presence of Nutlin-3 were not subject to turnover but were stable over 90 min (Figure $1 \mathrm{i}$ and Supplementary Figure 1A; Nutlin-3). Consistent with a mixed population of mono- and polyubiquitinated p53 (Figure 1e) the higher molecular weight forms in IR exposed cells were turned over, whereas the monoubiquitin-adducts persisted.

We next addressed the involvement of MDM2 in Nutlinstimulated p53 modification. First, we confirmed that although Nutlin-3 inhibits the high-affinity interaction between MDM2 and the Box-I domain of p53, it stabilizes binding of MDM2 to a weaker interaction site (Box-V) in the core domain (Figure 1j) that is essential for MDM2-mediated ubiquitination of p53. ${ }^{12}$ Furthermore, Nutlin-3 did not inhibit binding of endogenous p53 and MDM2 in cells. In fact, when p53 immunoprecipitates were analysed, there was a large fold increase in the amount of MDM2 found in complex with p53 ( 20-fold) in the presence of the drug, whereas the amount of p53 protein in the immune complex was modestly increased (Figure $1 \mathrm{k}$ ). To extend the immunoprecipitation data, proximity ligation assays (PLA; Figure 1I) were used to measure endogenous p53:MDM2 interactions in situ. Figure 11 (left panel) shows a low level of p53:MDM2 complexes in untreated cells with PLA foci distributed throughout the cell. However, in the presence of Nutlin-3, there was a significant increase in complex formation (Figure 11; right bottom panel) and this was primarily in the nucleus. To determine whether the increase in p53:MDM2 complex formation detected could result in MDM2-mediated ubiquitination of p53, we used the MDM2 inhibitor protein ARF. $^{22}$ ARF was chosen as a tool rather than MDM2 knockdown using siRNA because we were unable to prevent significant increases in MDM2 protein levels in response to Nutlin-3 under conditions where a $60-70 \%$ knockdown was achieved in the control cells (data not shown). In two different cell lines (Figure $1 \mathrm{~m}$ ), a titration of ARF could overcome the effect of Nutlin-3 on p53 ubiquitination. Studies showing that MDM2 is primarily a monoubiquitin ligase for $\mathrm{p} 53,{ }^{16}$ together with the data in Figures $1 \mathrm{~m}$ and $\mathrm{I}$, is consistent with the

Figure 1 Nutlin and IR induce stable monoubiquitination of p53. (a) Nultin-2 (yellow sticks) bound to the hydrophobic pocket of MDM2 (shown as surface); PDB: 1RV1. ${ }^{49}$ (b) Immunoblot of A375 cells treated with Nutlin-3 $(10 \mu \mathrm{M})$ or DMSO control for $8 \mathrm{~h}$, lysed in Triton-X buffer and developed using indicated antibodies. (c) Immunoblot of A375 cells treated with Nultin-3 $(10 \mu \mathrm{M})$ for $0-8 \mathrm{~h}$, lysed in urea buffer and developed using D01 to detect endogenous p53. (d and e) Immunoblot of His-ubiquitinated endogenous proteins isolated using Ni-NTA chromatography after A375 cells were transfected with His-ubiquitin $(0.5 \mu \mathrm{g})$ for $20 \mathrm{~h}$ and treated with (d) Nutlin-3 (10 $\mu \mathrm{M})$ or DMSO for the last $8 \mathrm{~h}$, or (e) untreated (lane 2) or treated with either DMSO (lane 3), Nutlin-3 (10 $\mu \mathrm{M}$, for last $8 \mathrm{~h}$; lane 4) or IR (5 Gy, with a $3 \mathrm{~h}$ recovery; lane 5). The cells were collected and lysed, the His-ubiquitinated proteome was isolated using Ni-NTA chromatography. The membrane was developed using antibodies to the indicated proteins. Cartoon in d: polyubiquitination in control cells will lead to proteasomal degradation, whereas the role of monoubiquitination is unknown. (f) Quantification of results from e using ImageJ. The fold increase of unmodified p53 and p53 attached to 1, 2 or 5 ubiquitins with respect to the control samples was calculated and is shown. (g) Immunoblot of A375 cells treated with Nutlin-3 $(10 \mu \mathrm{M})$, DMSO control or irradiated as above and simultaneously treated with lactacystin $(10 \mu \mathrm{M})$ for $4 \mathrm{~h}$, lysed in urea buffer and developed using D01. The IR samples were analysed on the same gel but lanes 3 and 4 were not adjacent (indicated by a line) to lanes 1 and 2. (h and i) Immunoblot of A375 cells treated with Nutlin-3 (10 $\mu$ M, 8 h; or DMSO control) or irradiated as in $\mathbf{d}$ and treated with cycloheximide $(30 \mu \mathrm{g} / \mathrm{ml}$ ) for the times indicated, lysed in (h) Triton-X buffer or (i) urea buffer and developed with DO1. The data from j were analysed by densitometry and depicted graphically. (j) Immobilized p53 Box-I (PPLSQETFSDLWKLLP) and Box-V (RNSFEVRVCACGRD) peptides incubated with MDM2 $(100 \mathrm{ng})$ and a titration of Nutlin-3 (0-8 $\mu \mathrm{M})$. Binding was detected using an anti-MDM2 mAb. (k) Immunoblot of p53 mAb immunoprecipitate from A375 cell lysate after Nutlin-3 $(10 \mu \mathrm{M})$ or DMSO treatment for $8 \mathrm{~h}$. (I) Left panel: PLA in A375 cells treated with Nultin-3 (10 $\mu \mathrm{M})$ for $8 \mathrm{~h}$ developed using anti-MDM2 mAb 4B2 and p53 pAb CM1 or BSA control. The data are representative of four separate experiments. Right top panel: cartoon of the PLA reaction where antibodies binding to MDM2 and p53 are detected by secondary antibodies conjugated to complementary DNA that come together to form a rolling circle PCR template (detected using fluorescent probes) when the two proteins are closer than $40 \mathrm{~nm}$. Right lower panel: PLA foci in at least 10 fields were counted; the average numbers of foci/cell are shown. ${ }^{*} P$-value $<0.01$. The $P$-value was calculated using Student's t-test. (m) A375 cells were transfected with a titration of p19ARF or empty vector (DNA normalized using empty vector). After $24 \mathrm{~h}$, the cells were treated with Nutlin or DMSO alone (as above) and collected $4 \mathrm{~h}$ later into urea lysis buffer. The lysates were analysed by immunoblot developed using antibodies to the proteins indicated. $(\mathrm{C}=\mathrm{control})$. $\mathrm{All}$ experiments represent the results of at least three independent experiments 
j
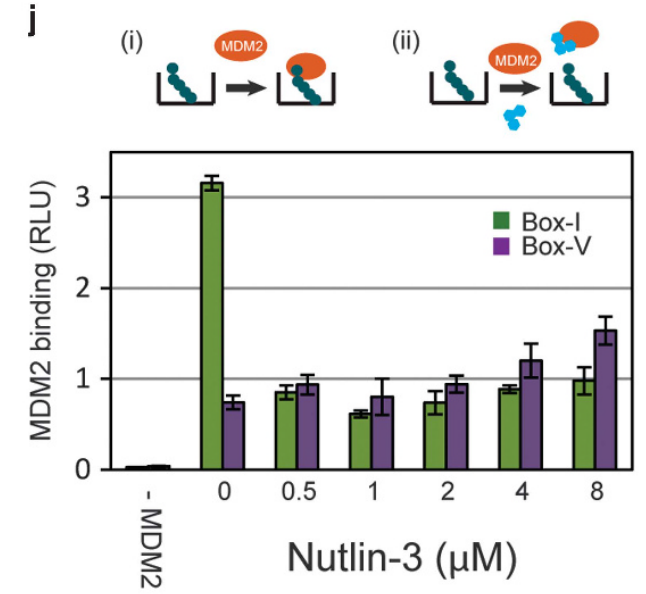

k

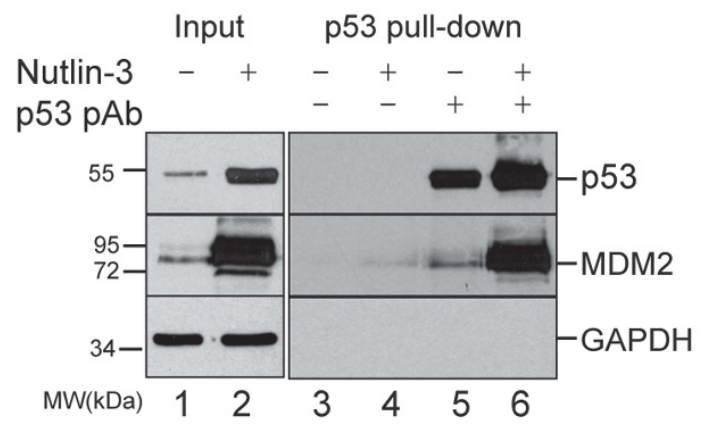

I
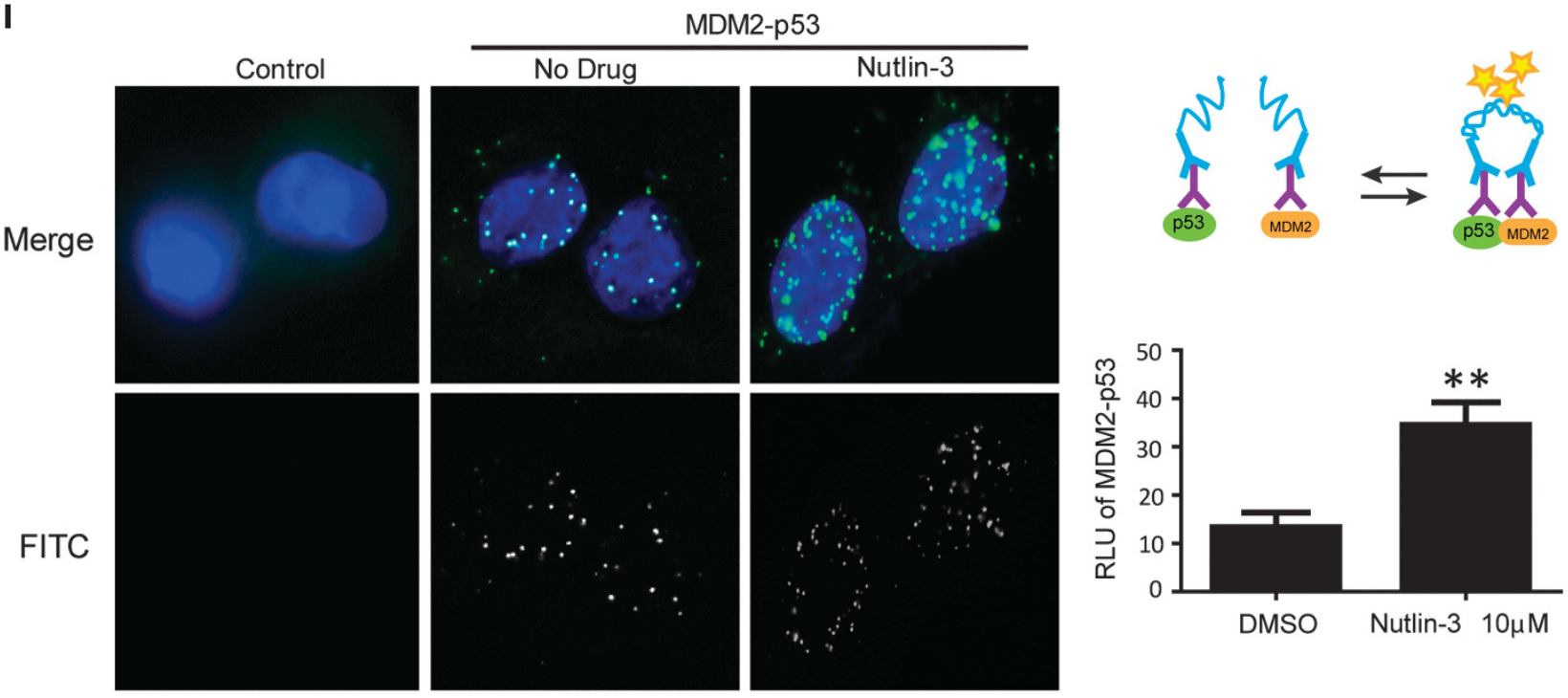

m

\section{HCT116}
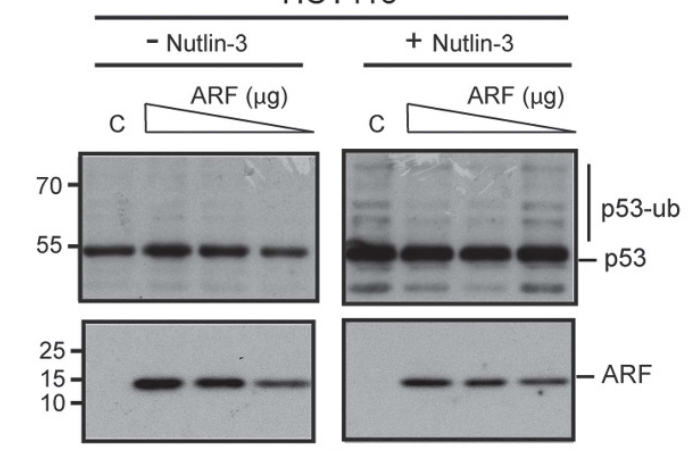

MW(kDa)
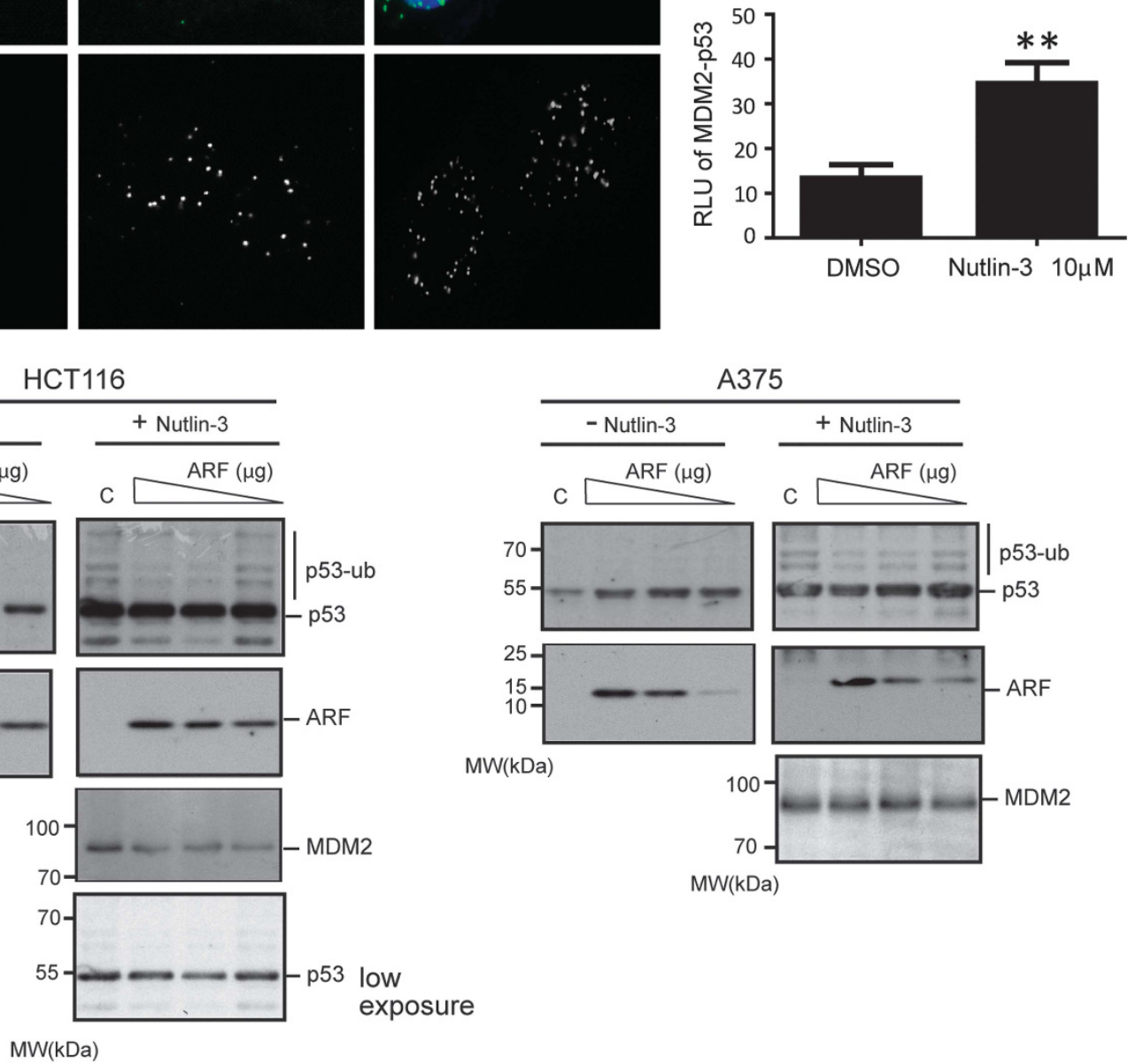

Figure 1 Continued

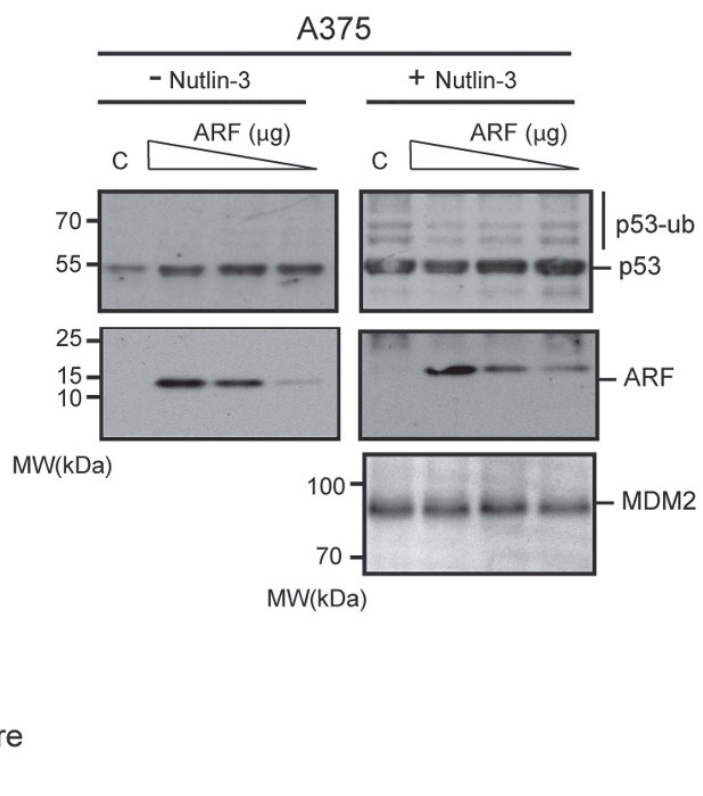


hypothesis that Nutlin-3 enhances p53 monoubiquitination through a process that involves MDM2.

Monoubiquitinated p53 is in the nucleus. Monoubiquitination has previously been proposed to signal p53 nuclear export and TA inhibition. ${ }^{23,24}$ However, such studies used C-terminal ubiquitin-fusions or overexpression systems. Here we found recapitulation of the p53-monoubiquitination pathway and its regulation by Nutlin-3 using exogenous components to be problematic, most likely reflecting the finely balanced nature of the pathway. To study the localization of the ubiquitinated forms of p53, we used PLA (Figure 2a and Supplementary Figure 3). When PLA assays were carried out in control cells, there was a background level of ubiquitinated p53 localized predominantly to the cytoplasm (Figure $2 \mathrm{~b}$ ) and no signal was detected in the control (Figure 2b) or in p53 null HCT116 cells (Supplementary Figure 2A). There was a fivefold increase in the number of positive p53:ubiquitin foci detected in the nucleus following Nutlin-3 treatment (Figures 2b, c and Supplementary Figure 3). In contrast, treatment with lactacystin induced PLA foci throughout the cells (Supplementary Figure 2B).

Data showing that monoubiquitinated p53 is located in the nucleus supports the hypothesis that it represents a step in the p53-activation pathway and suggests that the site-specific context of the ubiquitin-modification is likely to be a critical determinant of outcome.

\section{A pool of monoubiquitinated p53 is tightly associated} with the chromatin. We do not know if the nuclear pool of monoubiquitinated p53 is active for DNA-binding. To address this, the nucleus was separated into soluble and chromatinbound fractions. Figure 3 shows in Nutlin-treated cells a minor portion of the total ubiquitinated p53 (Figure 3a) was recovered in the cytoplasm (Figure 3b). However, when we analysed the two nuclear fractions, the majority of ubiquitinated p53 was chromatin-bound and ubiquitinated forms were below the level of detection in the soluble nuclear fraction. Although p53 protein levels were increased by Nutlin, HP1a, a nuclear marker, was consistently decreased in the soluble nuclear fraction of drug-treated cells. PARP, a marker for the soluble nuclear proteins, was unaffected by Nutlin-3 and Histone H3, a marker for the chromatin-bound fraction, showed a small but consistent increase in drugtreated cells. To confirm that the high molecular weight forms of p53 detected in the chromatin-bound fraction were generated by ubiquitination, hypotonic lysis was used to isolate chromatin-bound proteins (together with other insoluble proteins). The resultant pellet, which contained all the detectable DNA (Figure 3c) and the majority of histone $\mathrm{H} 1$, was resuspended and incubated with the p53deubiquitinating enzyme HAUSP. Under these conditions monoubiquitinated p53 could be reduced to a single nonubiquitinated form of the protein (Figure $3 d$ ). Thus, the nuclear pool of modified p53 detected by PLA (Figure 2) appears to be chromatin-bound and can be deubiquitinated.

Next a sucrose gradient sedimentation technique ${ }^{25}$ was adapted to determine the distribution of p53 in relation to the density of the chromatin. Nuclei isolated from control, Nutlin-3-

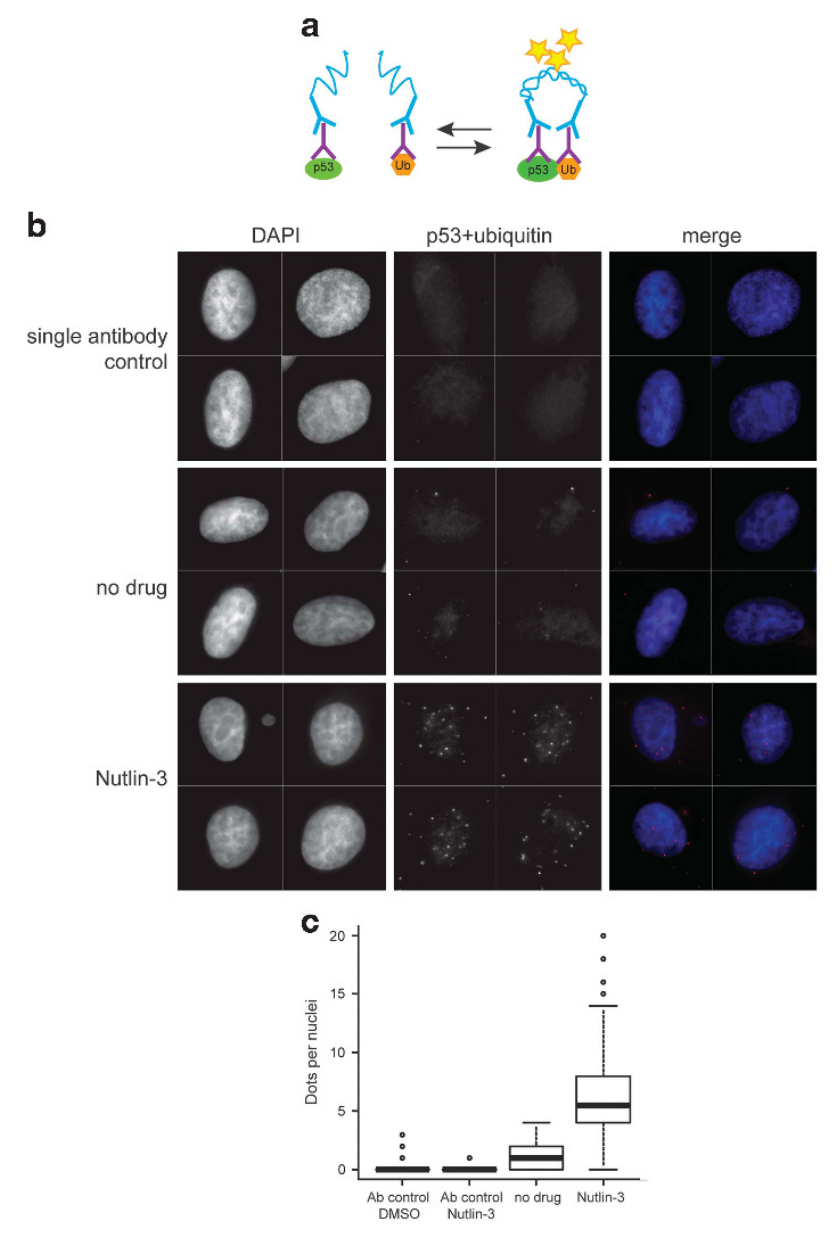

Figure 2 Monoubiquitinated endogenous p53 localizes to the nucleus. (a) Schematic illustration of the PLA as in Figure 3. (b) PLA in A375 cells treated with Nultin-3 $(10 \mu \mathrm{M})$ for $8 \mathrm{~h}$ using anti-ubiquitin only, or anti-ubiquitin and p53 D01 as indicated. (c) PLA foci in at least 100 cells were counted; the average numbers of foci/ cell are shown. The $P$-value was calculated using Student's $t$-test

and IR-treated cells were digested with micrococcal nuclease and analysed using an isokinetic 10/50\% sucrose-densitygradient (Figure 3e). The top one to three fractions of the gradient contain the chromatin-unbound nuclear protein and is where recombinant p53 sediments (Supplementary Figure 4A); fractions four to six contain loosely chromatinassociated proteins, whereas tightly chromatin-associated proteins are present in fractions seven to nine ${ }^{25,26}$ (Figure 3f). Analysis of IR- (Figure 3g) or Nutlin-3 (Figure 3h)-treated cells showed no pronounced shift in the distribution of total p53 compared with control cells; however, there was a striking appearance of mono- and multi-monoubiquitinated forms in the tightly chromatin-associated fractions. The data indicate that the (multi-)monoubiquitinated pool of p53 is in an active tightly chromatin-associated state. Note that chromatin fractionation is carried out under non-denaturing conditions and the pan de-ubiquitinase inhibitor NEM cannot be used (NEM inhibits p53 DNA-binding; Supplementary Figure 4B); therefore, the portion of p53 in a monoubiquitinated form is likely to be an underestimate. 
a
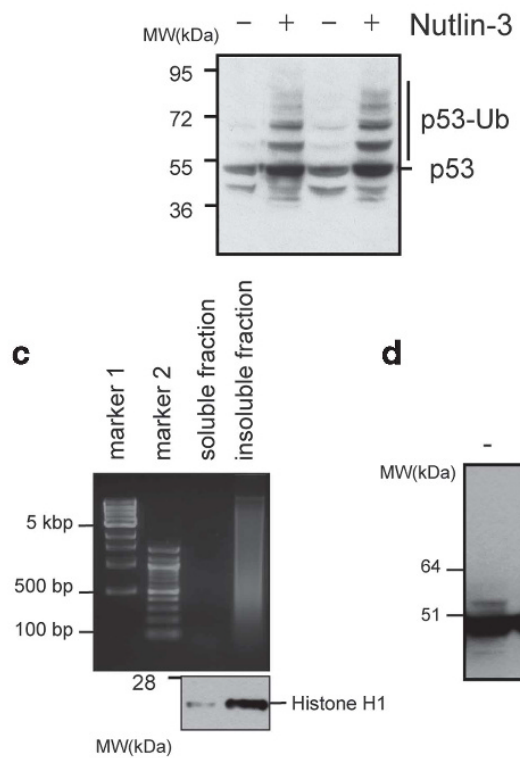

e

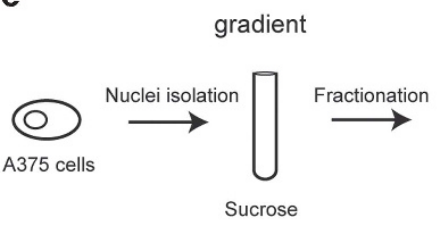

g

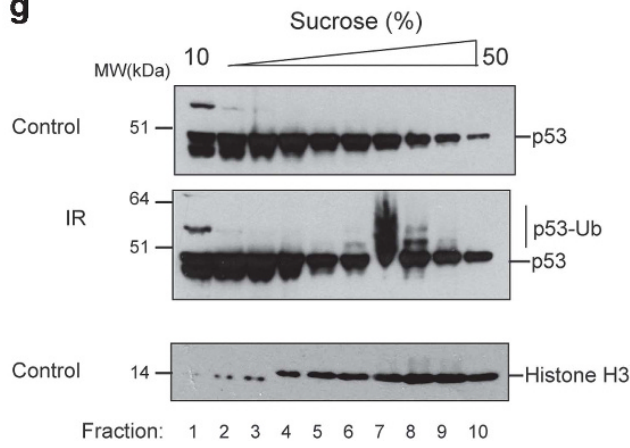

b
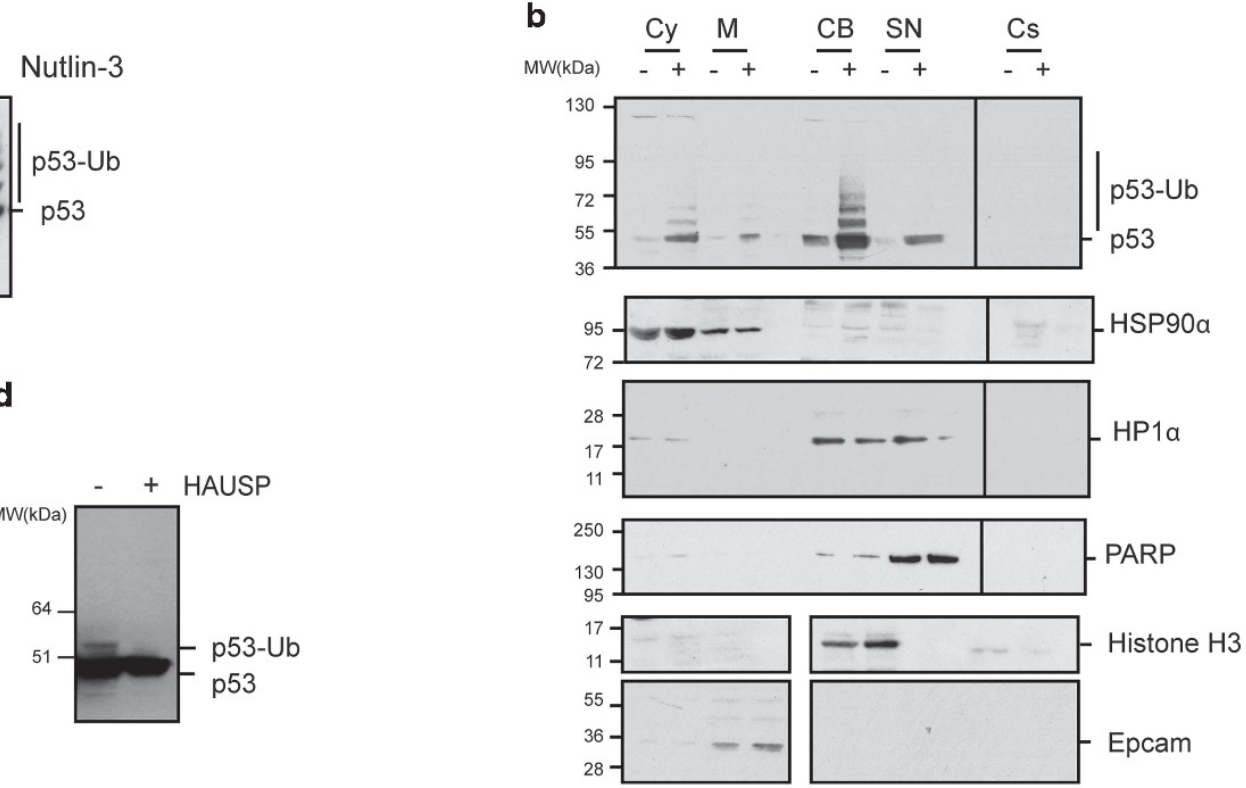

f

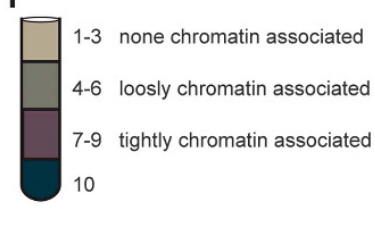
MW(kDa)

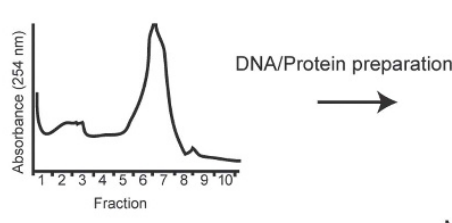

h
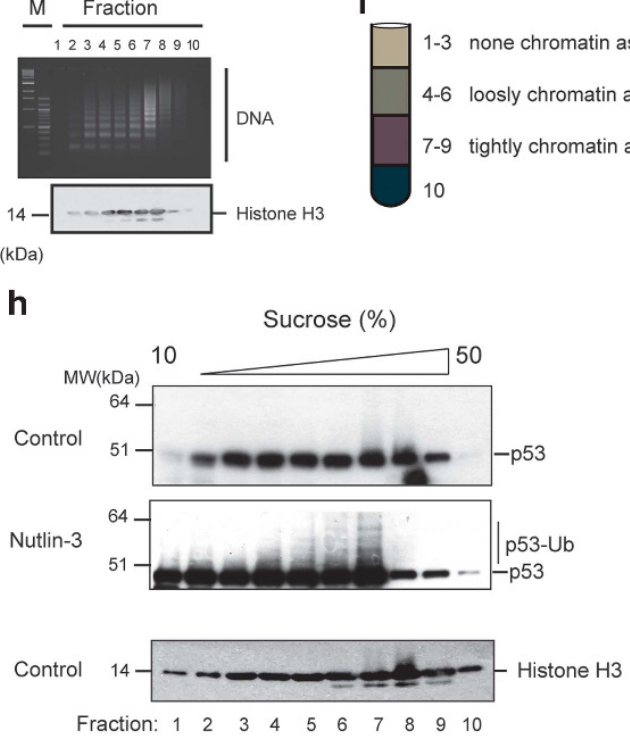

Figure 3 A pool of monoubiquitinated p53 is tightly associated with the chromatin. (a) Duplicate plates of A375 cells were treated with carrier (DMSO) or Nutlin-3 (10 $\mu \mathrm{M})$ for $8 \mathrm{~h}$ before collecting and lysed in urea lysis buffer. The whole cell lysates were analysed using SDS-PAGE followed by immunoblot developed using anti-p53 mAb D01. (b) A375 cells were prepared as in a and fractionated using a Subcellular Protein Fractionation kit into cytoplasmic (Cy), membrane (M), chromatin-bound (CB), soluble nuclear (SN) and cytoskeletal (Cs) fractions. The fractions were analysed by SDS-PAGE and immunoblot; proteins were detected using antibodies to the indicated proteins. The black lines mean that the samples were analysed at the same time and immunoblotted together but were not adjacent on the gel. Where there is a gap samples were analysed using the same equipment but on separate gels (in this case, markers were used to ensure normalization of electrophoresis and transfer). The data are representative of duplicates from two separate experiments. (c) Cells were lysed under hypotonic conditions and the insoluble and soluble fractions collected. DNA content was determined using chloroform-methanol extraction and analysed on an agarose gel, whereas protein was analysed by SDS-PAGE/immunoblot and histone H1 detection. (d) Irradiated cells (5 Gy) were lysed to isolate insoluble proteins as in c. The pellet was resuspended and incubated in the present or absence of HAUSP for $30 \mathrm{~min}$ at $30^{\circ} \mathrm{C}$ before analysis by SDS-PAGE/immunoblot and detection of p53 using D01. Unmodified (p53) and ubiquitinated p53 (p53-Ub) are indicated. (e) Experimental outline of nuclei fractionation using sucrose gradient centrifugation. A375 cell nuclei were isolated, RNA removed and chromosomal DNA partially digested using micrococcal nuclease. Separation was performed using a 10-50\% sucrose gradient and fractions $(0.5 \mathrm{ml})$ collected for analysis. A UV trace and agarose gel of the DNA content are shown, plus an immunoblot for histones. (f) Schematic illustration of distribution of chromatin and chromatin-associated protein in the sucrose gradient. (g and h) Nuclei of control or irradiated (G; 5 Gy, $3 \mathrm{~h}$ recovery) and Nutlin-3 (H; $10 \mu \mathrm{M}$ for $8 \mathrm{~h}$ ) treated A375 cells fractionated as described in $\mathbf{e}$ and analysed by immunoblot using DO1 and Histone $\mathrm{H} 3$ antibodies. The data are representative of at least two individual experiments

A direct effect for p53 ubiquitination on its DNA-binding activity. p53 tetramers, purified from insect cells, can be separated into DNA-binding latent or activate fractions. ${ }^{27}$ The DNA-binding active fraction of p53 was used to determine the effect of ubiquitin on binding to short oligonucleotide probes (Figure $4 \mathrm{~b}$ and Supplementary Figure S5). p53 was ubiquitinated in vitro by MDM2 or incubated in control reactions (Figure 4a). Though MDM2 is published to be a monoubiquitin 

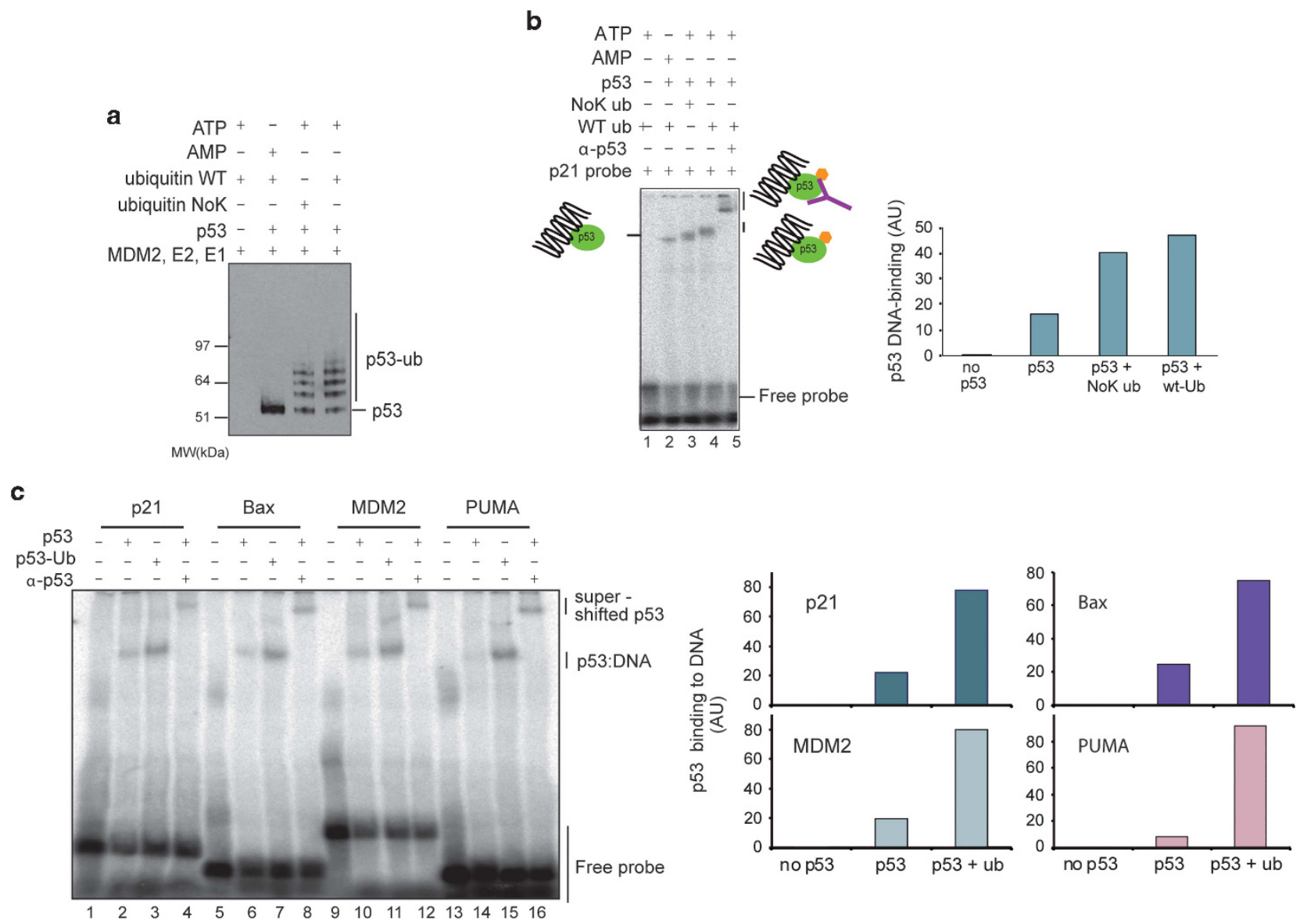

d

2283-AGCATTGGCTTTCTGGCCATCAGGAACATGTCCCAACATGTTGAGCTCTGGCATAGAAGAGGCGCT-2342
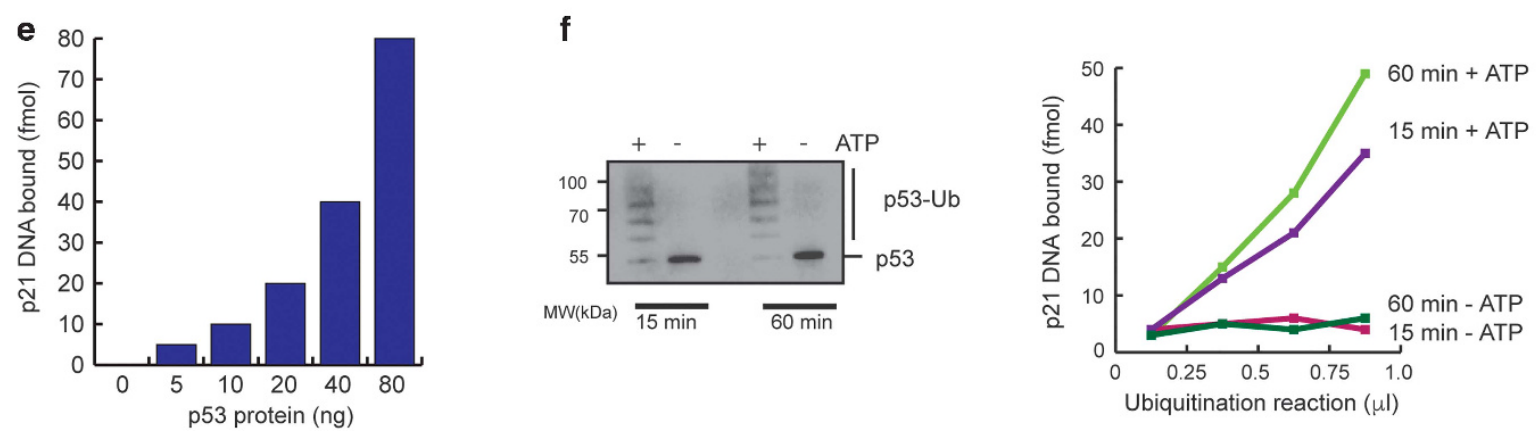

Figure 4 DBD ubiquitination can contribute directly to p53:DNA binding. (a) p53 was ubiquitinated for 45 min with either wt or mutant ubiquitin (NoK). (b) Binding of modified p53 (500 ng; from a) to p21-oligonucleotides was measured in an EMSA. Where indicated, D01 was added to supershift the p53:DNA complex. The control was p53 incubated in the ubiquitination reaction plus AMP in place of ATP to prevent ubiquitination. Bands were quantified using ImageJ and are presented graphically (b, right panel). (c) As in $\mathbf{b}$, except that a range of p53-binding oligonucleotides based on the indicated promoters were used. The control reactions (p53) were incubated in the ubiquitination reaction plus AMP in place of ATP to prevent ubiquitination. Results are representative of at least two independent experiments. (d) Nucleotide sequence of the DNA fragment used to assay p53 DNA-binding. The p53 binding element is shown in blue/red. (e) p53 (purified from Sf21 cells) binding to the radiolabelled probe shown in $\mathbf{d}$ was determined using a filterbinding assay. The graph shows fmols of DNA bound per ng of p53. (f) Immunoblot p53 (detected using D01) ubiquitinated by MDM2 in the presence or absence of ATP incubated for either 15 or $60 \mathrm{~min}$ (left panel). p53 from the ubiquitination reactions shown was subsequently assayed for DNA binding as in e (right panel). The results are indicative of three separate experiments

ligase ${ }^{14}$ we used mutant Ub (NoK; Lys residues are mutated to $\mathrm{Arg}$ ) to ensure that no polyubiquitination of p53 took place (Figures $4 a$ and b). The ability of p53 to bind a 33 bp element from the $21^{\text {WAF1 }}$ promoter was increased approximately threefold by ubiquitination and monoubiquitination was sufficient to stimulate activity. In addition, ubiquitin had to be covalently attached to the TA as no enhancement was seen in control reactions containing ubiquitin and other assay 
components but no ATP (Figure 4b). To determine whether the effect of ubiquitination on p53 activity was specific to the p2 $1^{\text {WAF1 }}$ promoter probes with the $\mathrm{p} 53$ responsive elements from $B A X, M D M 2$ and PUMA were used (Figure 4c). Figure $4 c$ shows that increased DNA-binding for ubiquitinated p53 was seen in all the cases (three- to fourfold). Using a rapid filter method, we developed a quantitative stopped DNA-binding assay for p53 on a longer 66 bp fragment ${ }^{28}$ (Figure 4d). Using conditions where there was a linear relationship between the amount of p53 and the amount of labelled DNA bound (Figure $4 \mathrm{e}$ ), we observed that ubiquitination strongly enhanced binding of p53 to the longer fragment (Figure 4f). Thus, ubiquitination enhanced p53 activity on both short oligonucleotide and longer DNA fragments.

a
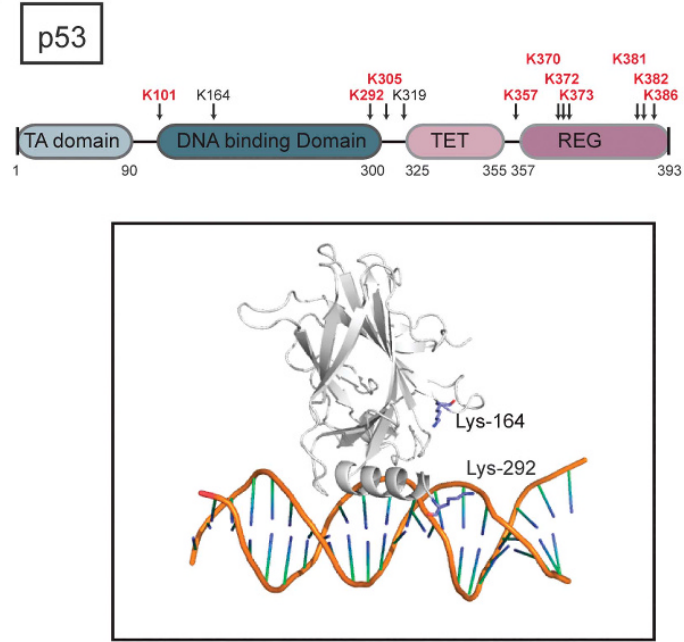

b

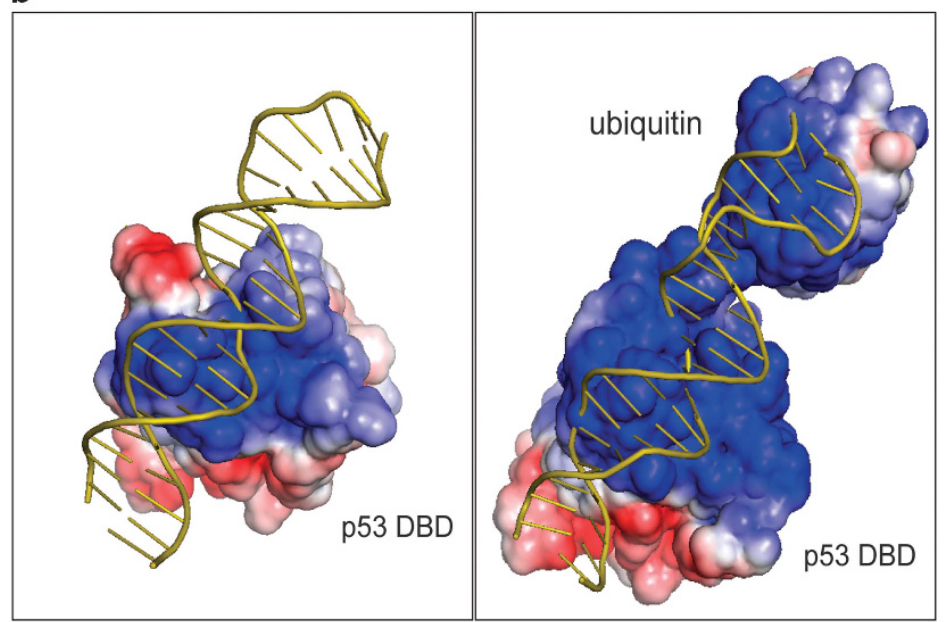

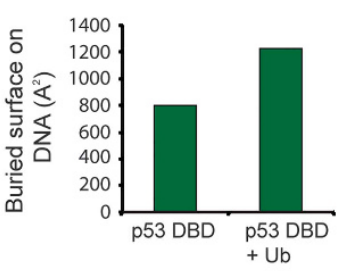

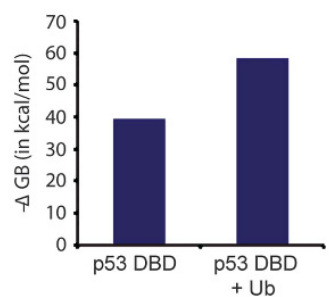

Figure 5 MD simulations are supported by increases in p53 DNA-binding activity. (a) Domain and crystal structure (DBD only, pdb:1TUP) of p53 with Lys that are subject to ubiquitination by MDM2 (red) and/or Pirh2 indicated. ${ }^{50}$ (b) Model of the p53:ubiquitin:DNA complex. Electrostatic surface analysis of the ubiquitin:TA model used the APBS Pymol plugin. Blue indicates regions of positive potential, while red indicates regions of negative potential. (c) The DNA surface buried by the p53-1 DBD alone or in complex with ubiquitin was calculated using Pymol (upper panel). Molecular dynamic simulations were carried out on the complex of p53 DBD:DNA (PDB:1TUP) and the p53 DBD:DNA: ubiquitin model. The simulations were used to compute the free binding energy (in $\mathrm{kcal} / \mathrm{mol}$ ) of DNA with p53 DBD \pm ubiquitin (lower panel)

Figure 6 DBD ubiquitination directly increases the DNA-binding ability of the TA IRF-1. (a) Domain and crystal structure of IRF-1 (DBD only, pdb: 1IF1) with Lys that are subject to ubiquitination indicated. (b) In vitro ubiquitination reactions with CHIP, IRF-1 plus wt or mutant (NoK) ubiquitin were incubated for 45 min. The control was IRF-1 incubated in the ubiquitination reaction plus AMP in place of ATP to prevent ubiquitination. (c) Binding of IRF-1 (from b) to C1 oligonucleotide probe in an EMSA (100 ng IRF-1/ lane), bands were quantified using ImageJ and are presented graphically (lower panel). (d) Binding of IRF-1 (from b) to C1 oligonucleotide immobilized on a microtiter well incubated with increasing amounts of IRF-1 (0-40 ng, from b) in the mobile phase. Binding was detected using anti-IRF-1 mAb. Results shown are representative of three independent experiments. (e) Model of the IRF-1:ubiquitin:DNA generated by superimposing IRF-1:ubiquitin onto the IRF-1 DBD:DNA crystal structure. Electrostatic surface analysis of the ubiquitin:TA model used the APBS Pymol plugin. Blue indicates positive charge, while red shows negative charge. (f) The DNA surface buried (upper panel) by the IRF-1 DBD alone or in complex with ubiquitin and the free binding energy (lower panel) were calculated as in Figure $5 \mathrm{c}$ and are shown 
DBD ubiquitination can contribute directly to p53:DNA binding. Following pathway activation, stably monoubiquitinated p53 is in the nucleus where it is tightly associated with

a

IRF-1

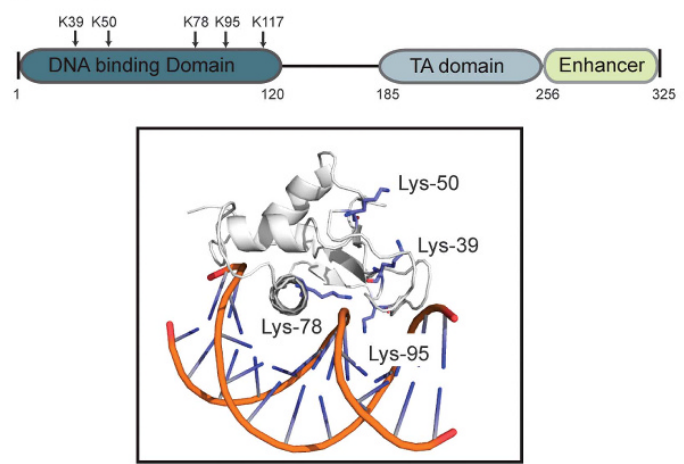

C
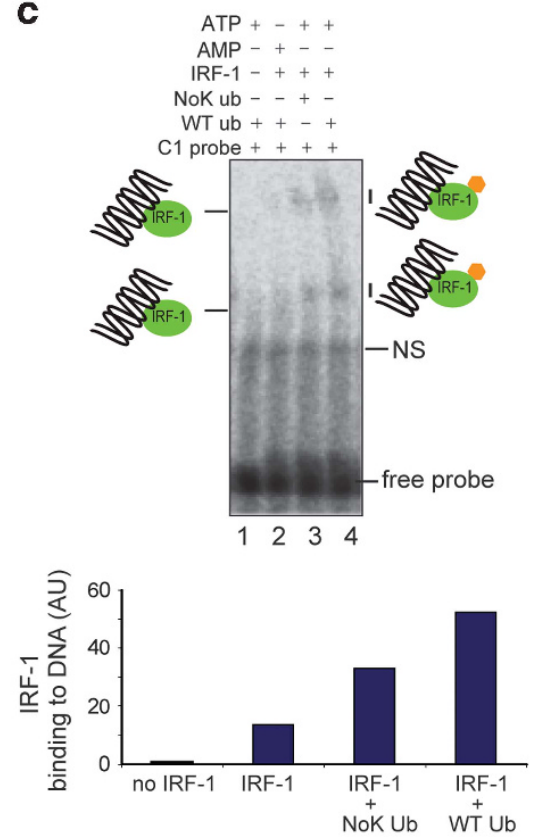

chromatin; furthermore ubiquitination directly enhances the interaction of p53 with DNA (Figures 3 and 4). Next, we addressed the mechanism by which ubiquitin affects p53 binding

b
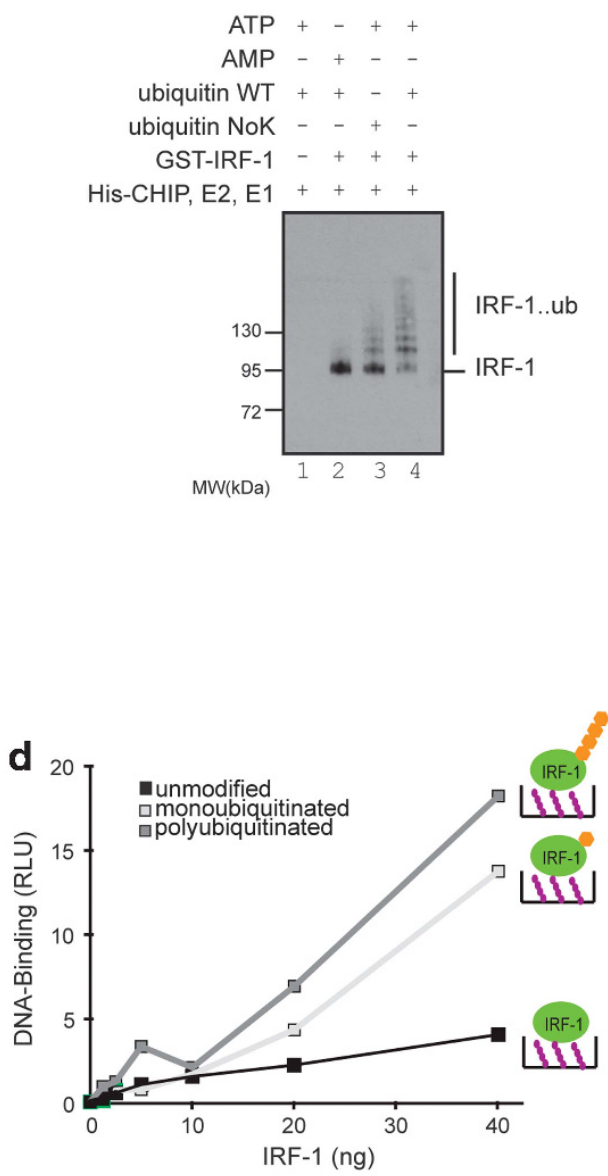

e
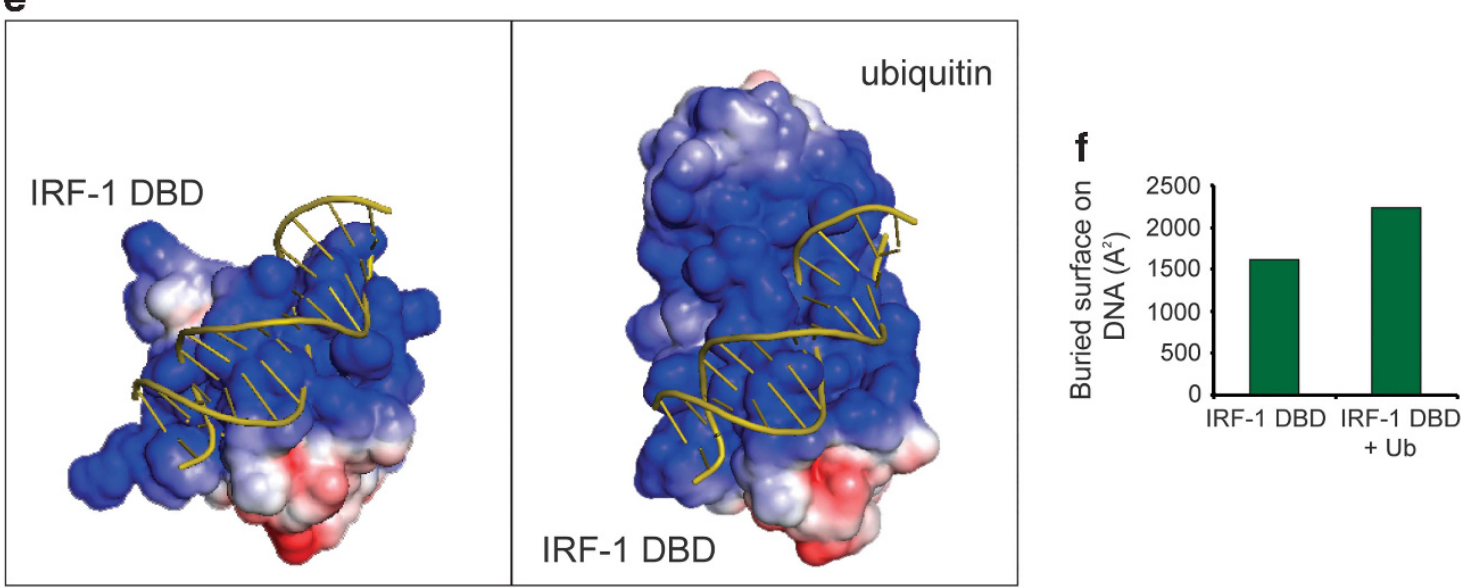
to DNA. We hypothesized that ubiquitin-acceptor lysine residues located in the DBD of $\mathrm{p} 53^{10}$ were of most interest and in order to predict the impact of monoubiquitination on
p53:DNA-binding we used in silico modelling. First, a model in which Lys ${ }^{292}$ or Lys ${ }^{164}$ was modified by the C-terminal glycine of ubiquitin was generated using the human p53 DBD
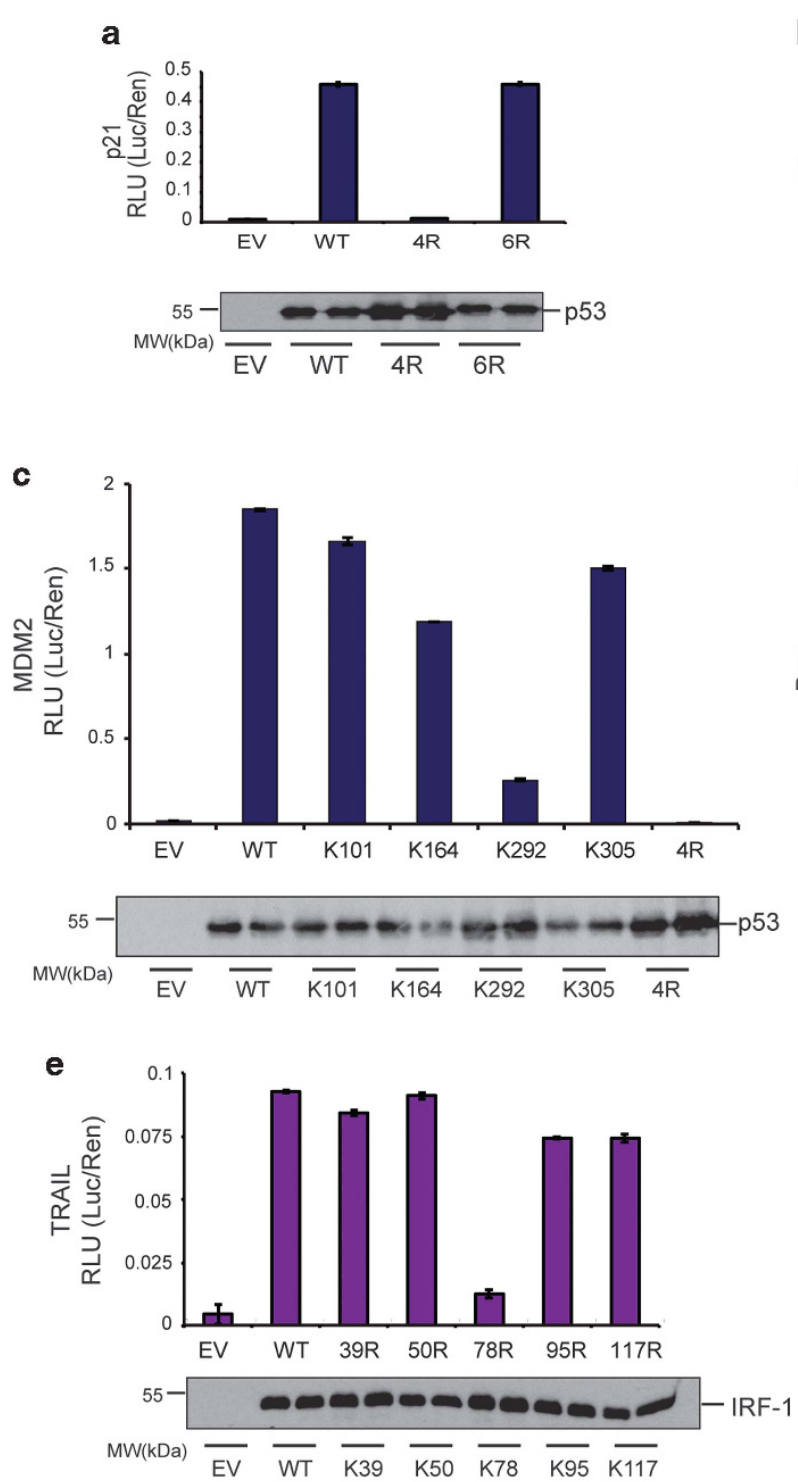

g

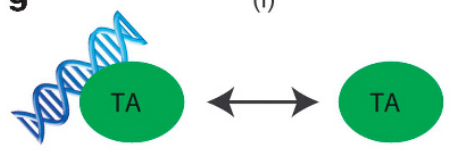

b

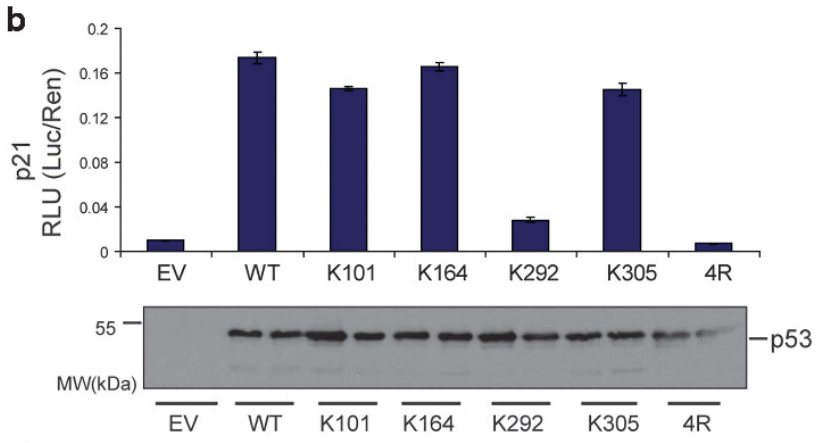

d
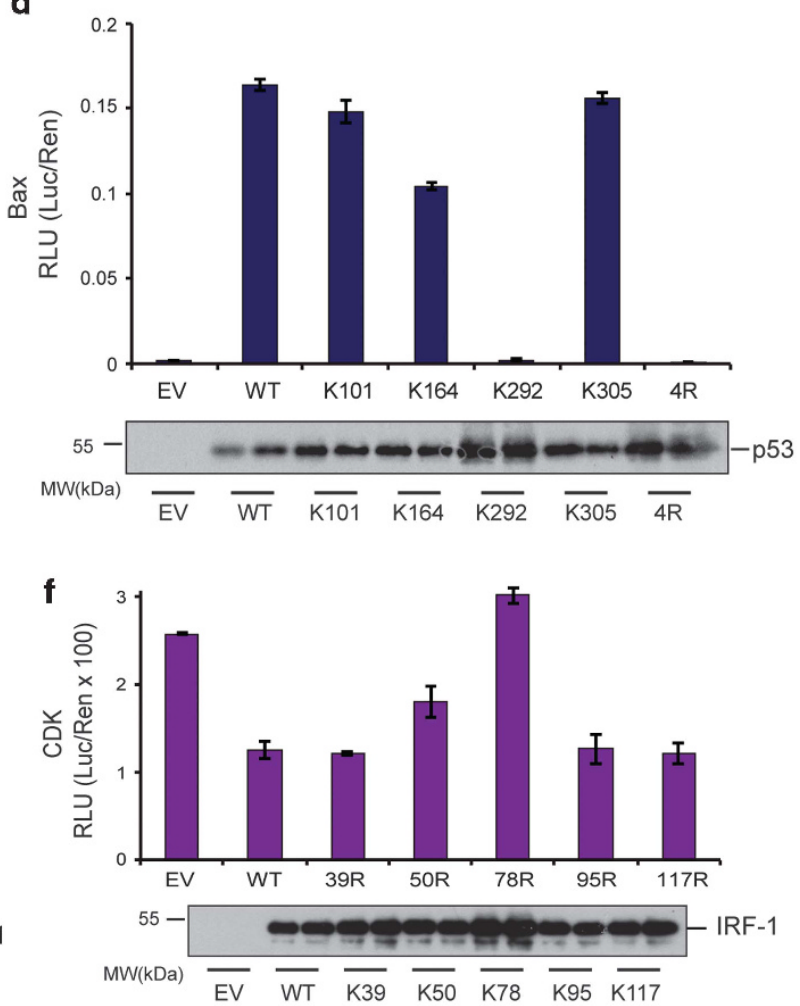

(ii)

(iii)
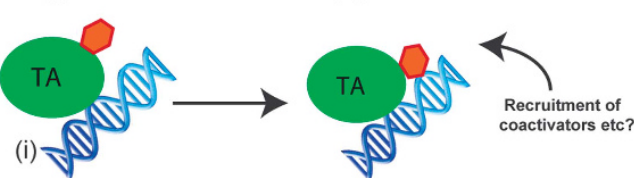

(iv)

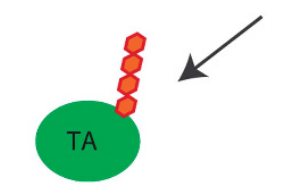


(PDB:1TUP) and ubiquitin (PDB: 1UBQ) structures and the HADDOCK webserver (Supplementary Figures 6 and 7). The resulting models provided a starting structure for molecular dynamic (MD) simulations in an explicit solvent system. During the simulation, ubiquitin underwent conformational changes that brought it into close proximity with the DNA, stabilizing intermolecular interactions (Figure 5b and Supplementary Movie 1). Ubiquitin occupied a similar position in relation to the p53 DBD when attached at K164 or K292 (Supplementary Figure 7); as only K292 is targeted by MDM2, ${ }^{8}$ we decided to focus on this site. Electrostatic surface analysis of the complex using APBS (Adaptive Poisson-Boltzmann Solver) in Pymol, shows that addition of ubiquitin to the DBD extended the positively charged surface area that forms the protein:DNA interface (Figure 5b). In addition, analysis of the DNA surface buried by the protein increased $53 \%$ when the DBD was monoubiquitinated (Figure $5 c$ ). Further, when the simulations were used to calculate binding energies between DNA and the p53 DBD, a significant decrease in $\triangle \mathrm{GB}$ of monoubiquitinated compared with unmodified p53 was predicted (Figure $5 \mathrm{c}$ ).

The in silico experiments are intriguing as they suggest a novel role for monoubiquitin in direct promotion of stable TA: DNA interactions, where specific TA DBD:DNA interactions are enhanced by nonspecific ubiquitin:DNA binding.

DBD ubiquitination directly increases the DNA-binding ability of the TA IRF-1. p53 has multiple ubiquitin-acceptor sites and the isolated DBD is not a substrate for MDM2 making it difficult to separate DBD ubiquitination from other p53 ubiquitination events. To complement our studies on p53, we therefore used IRF-1, an interferon-regulated TA that is ubiquitinated exclusively in its $\mathrm{DBD}^{29}$ (Figure 6a). IRF-1 ubiquitination occurs at several DBD sites in close proximity to the DNA leading to speculation that it might affect IRF-1 DNA-binding activity. ${ }^{29}$ Following the modification of IRF-1 with either wt or NoK ubiquitin (Figure 6b), there was a significant gain in TA DNA-binding (Figures $6 \mathrm{c}$ and d). Models of monoubiquitinated DBD were generated as for $p 53^{30}$ and MD simulations show a similar increase in buried DNA surface and significant decrease in $\triangle G B$ (Figures $6 e$ and f). Thus, studies on IRF-1 support the concept that DBD monoubiquitin is sufficient to increase TA sequence-specific DNA-binding activity.

Key DBD lysine residues are required for maximal TA activity in cells. If the increase in DNA-binding activity and enhanced chromatin association seen on monoubiquitination of p53 result in a gain of TA function, we would expect that loss of key acceptor Lys residues would adversely affect the
TA activity of $\mathrm{p} 53$ and IRF-1. A series of conservative mutations (Lys to Arg) were introduced at known ubiquitinacceptor residues within, or adjacent to, the DBDs of p53 and IRF-1 (Figures 5a and 6a). First, the activity of a p53 mutant in which four DBD ubiquitin-acceptor sites were mutated (4R) was compared with one where six C-terminal domain acceptor sites were substituted (6R). Although the $6 \mathrm{R}$ retained wild-type p53 activity levels, 4R was essentially transcriptionally inactive (Figure $7 \mathrm{a}$ ). To pinpoint which of the Arg substitutions contributed to the loss of function, individual point mutant proteins were examined (Figures $7 b-d)$. In agreement with the computational analysis, the K292Rmutant consistently demonstrated the most significant decrease in activity though it retained the ability to bind DNA (Supplementary Figure 8). Thus, the loss of transcriptional activity seen in the K292R-mutant supports the idea that post-translational modification at this site might be involved in regulating p53 TA function.

Interestingly, K164R displayed a significant reduction in TA activity using MDM2- or BAX-reporters, but not on the p21reporter suggesting that there could be some promotercontext specificity with respect to modification at a given DBD site. When similar experiments were carried out using IRF-1 with point mutations at each of five potential ubiquitinacceptor residues, only the K78R mutation consistently produced a decrease in both the TA (Figure 7e) and repressor (Figure 7f) activity.

\section{Discussion}

Though recently we have gained a greater appreciation of how both nonproteolytic and proteolytic ubiquitin-pathways are used to regulate gene expression, there remains a significant lack of insight into the molecular mechanisms by which nonproteolytic modification, particularly monoubiquitination, regulates TA function. ${ }^{4}$ Here we explore why p53 activation by Nutlin-3 or IR is accompanied by increased ubiquitination. ${ }^{11,12}$ This apparent contradiction is resolved by demonstrating an unexpected function for ubiquitin as an enhancer of p53 activity through a direct contribution to the TA:DNA-binding interface (Figure 7g).

Artificial systems have revealed that ubiquitination of LexAVP16 can promote transcriptional activity and that the E3ligase component can be circumvented by fusing ubiquitin to the $\mathrm{N}$ terminus. ${ }^{31}$ Several studies have demonstrated that ubiquitin-fusions of, for example, FOXO4 or TAT, can modulate TA activity in mammalian cells. ${ }^{32,33}$ Though ubiquitin-fusion protein studies have been instrumental in demonstrating a link between monoubiquitin and TA function, we must be cautious in their interpretation as they are unlikely to provide effective

Figure 7 Mutation of the ubiquitin-acceptor lysines in p53 and IRF-1 DBD decrease their transcriptional activity. (a) H1299 cells were transfected with wt or Lys $\rightarrow$ Arg mutant p53 constructs (100 ng) plus p21-Luc (140 ng) and control Renilla-Luc $(60 \mathrm{ng})$. $6 \mathrm{R}$ has all six C-terminal ubiquitination sites mutated and 4R has all four ubiquitination sites in or proximal to the DBD mutated. Post transfection $(24 \mathrm{~h})$, the cells were collected and dual luciferase reporter assays performed. Results were normalized by expressing firefly/renilla luciferase activity in relative light units (RLU) as the mean \pm S.D. The cell lysates were analysed by SDS-PAGE/lmmunoblot. (b-d) H1299 cells were transfected with either wt or mutant p53 (Lys $\rightarrow$ Arg point mutations at Lys ${ }^{101}$, Lys ${ }^{164}$, Lys ${ }^{292}$, Lys ${ }^{305}$ or 4R) and assayed as in a with luciferase reporter plasmids for p21 (b), MDM2 (c) or Bax (d). (e and f) H1299 cells were transfected with wt or Lys $\rightarrow$ Arg mutant IRF-1 constructs (100 ng) for Lys, ${ }^{39}$ Lys, ${ }^{50}$ Lys $^{78}$, Lys ${ }^{95}$ or Lys ${ }^{17}$ and assayed as above with luciferase reporters for TRAIL (e) or CDK (f). (g) Model of a novel role of monoubiquitination in the activation of transcription 
tools to study the impact of site-specific modification. To illustrate this, fusion of ubiquitin to the C-terminal regulatory domain of p53 leads to its nuclear export. ${ }^{23,24}$ In contrast, endogenous p53, monoubiquitinated in response to activating signals, accumulates in the nucleus (Figure 2) and binds chromatin (Figure 3). Thus, we need to develop alternative approaches to explore physiological responses in a site- and signal-specific context. Site specificity is exemplified by the coactivator SCR-3, which is monoubiquitinated at two sites close to an LXXLL coregulator signature motif. ${ }^{34}$ Mutation of the Lys acceptor sites to Arg inhibits nuclear receptor binding to the LXXLL, whereas monoubiquitination stimulates SCR-3 function. Thus studies on context and site specificity of ubiquitination are likely to provide greater access to a mechanistic understanding of how signals lead to changes in gene expression.

An elegant series of experiments focused on the yeast $a 2$ provides one mechanism by which ubiquitin can modulate transcription. In this case, ubiquitination aids in the disassembly of transcription complexes from active promoters, a process that appears to be essential for robust derepression of a2 target genes. ${ }^{35}$ An alternative mechanism is proposed for GAL4 where monoubiquitination prevents it from being 'stripped' off the DNA by components of the proteasome. ${ }^{30}$ This observation led to the suggestion that monoubiquitin might 'clamp' GAL4 onto the chromatin. ${ }^{36}$

We have provided evidence for a novel interaction mode where ubiquitination at specific DBD Lys residues brings the charged surface of ubiquitin into a position where it can contribute directly to TA activity by expanding the DNA-binding interface; this results in an increased buried DBD:DNA surface and a decrease in free energy for the interaction (Figures 5 and 6). Thus weak non-covalent ubiquitin interactions with DNA may act as a 'clutch' to enhance promoter residency and productive promoter output rather than rapid turnover or 'treadmilling' of TAs. ${ }^{37}$ Alternatively, monoubiquitination may favour one mode of p53:DNA binding over another. Recent studies suggested that efficient function of p53 requires at least two modes of operation; a search mode mediated by the C-terminal domain that is characterized by largely nonspecific DNA binding coupled to fast-sliding, and a recognition mode in which p53 binds in a sequence-specific manner and can 'hop' on and off the DNA but is unable to slide. ${ }^{38}$

The data presented here suggest that $\mathrm{p} 53$ can be regulated by ubiquitination in a manner that requires ubiquitin to have a direct role in DNA binding. This model is supported by data showing that ubiquitin can bind weakly to DNA in its unconjugated state. ${ }^{39}$ Further, our model would leave the hydrophobic binding face of ubiquitin ${ }^{40}$ available for the potential recruitment of additional factors to further stabilize the TA:chromatin complex. In conclusion, site-specific monoubiquitination may impact on both the localization and function of p53.

\footnotetext{
Materials and Methods

Cell culture, transfection, half-life determination, reporter assays and immunoblotting. A375 and H1299 cells were maintained in DMEM and RPMI medium (Invitrogen, Carlsbad, CA, USA) supplemented with 5\% (v/v) FBS (Biosera, Boussens, France), 1\% (v/v) penicillin/streptomycin (Invitrogen), respectively, and grown at $37^{\circ} \mathrm{C}$ with 10 or $5 \% \mathrm{CO}_{2}$, respectively. Cell lysis was
}

performed as described previously. ${ }^{12}$ At $80 \%$ confluence, the cells were transfected using Attractene (Qiagen, Germantown, MD, USA) following the manufacturer's instructions. Reporter assays were carried out using the Dual Luciferase Reporter System from Promega (Madison, WI, USA) following the supplier's instructions. Half-life determination was carried out as published. ${ }^{41}$ The 4-12\% NuPAGE gels in a Mops buffer system (Invitrogen), run with either SeeBlue or Page Ruler Pre-Stained protein ladder (Thermo Fisher Scientific, Waltham, MA, USA), were analysed by immunoblots as described previously. ${ }^{20}$

Reagents, plasmids and protein preparation. Antibodies were, DO1 mAb and CM1 pAb (anti-p53; Moravian Biotechnology, Brno, Czech Republic), 4B2 mAb (anti-MDM2; Moravian Biotechnology), anti-ubiquitin mAb (Santa Cruz Biotechnology, Santa Cruz, CA, USA), anti-p21 mAb (Calbiochem, Darmstadt, Germany), anti-GAPDH mAb and anti-Histone-3 pAb and -Epcam pAb (Abcam, Cambridge, UK), anti- $\beta$-actin mAb (Sigma-Aldrich, St. Louis, MO, USA), antiHSP90 $\alpha$ (Enzo Life Sciences, Exeter, UK), anti-HP1 $\alpha$ and -PARP mAb's (Millipore, Billerica, MA, USA) and anti-IRF-1 mAb (BD Biosciences, San Jose, CA, USA). Secondary antibodies were from Dako (Glostrop, Denmark). Antibodies were used at the concentrations indicated by the supplier. IRF-1 and p53 mutants were constructed using a QuikChange site-directed mutagenesis kit (Stratagene, Agilent Technologies, La Jolla, CA, USA) with primers designed for a codon change from Lys to Arg (Sigma). GST-IRF- 1 and GST-MDM2 were purified using glutathioneSepharose and the tag was removed using Prescission Protease following the manufacturer's instructions (GE Healthcare, Pittsburgh, PA, USA). His-CHIP and His-UbcH5 were purified using Ni-NTA agarose (Qiagen) following the supplier's instructions. His-USP7 (HAUSP) was supplied by Boston Biochem (Cambridge, MA, USA). Untagged p53 purified from Sf9 cells was a gift from Dr Jennifer Fraser (University of Edinburgh).

Ubiquitination assay. In vitro ubiquitination assays were carried out using our published method, ${ }^{12}$ with $25-250 \mathrm{ng}$ of substrate [p53 or GST-IRF-1] and up to $60 \mathrm{ng}$ MDM2 or His-CHIP, as indicated. The reactions were incubated at $30^{\circ} \mathrm{C}$ for 10-45 min as indicated. In cell ubiquitination assays were carried out as published ${ }^{12}$ using A375 cells without MG132 treatment.

Proximity ligation assay. The cells grown on glass cover slips to $50 \%$ confluency were treated (as indicated), fixed by the addition of formaldehyde solution ( $4 \%$ (v/v) formaldehyde, $100 \mathrm{mM}$ PIPES (pH), $25 \mathrm{mM}$ EDTA, $1 \mathrm{mM} \mathrm{MgCl}$ ) for $15 \mathrm{~min}$ and permeabilized using $1 \%$ Triton X-100 in PBS for 10 min. Duolink II assays (Olink Bioscience, Uppsala, Sweden) were carried out following the supplier's instructions. The fluorescent signal was imaged using an Axioplan2 (Zeiss, Göttingen, Germany) fluorescent microscope.

Fractionation of cells and chromatin. Nuclei isolation, digestion and lysis was carried out as previously described, ${ }^{25}$ all solutions were supplemented with $20 \mathrm{mM}$ DTT. Soluble chromatin was fractionated using $10-50 \%$ (w/v) isokinetic sucrose gradients in TEEP80 (10 mM Tris- $\mathrm{HCl}, \mathrm{pH} 7.6,0.1 \mathrm{mM}$ EDTA, $0.1 \mathrm{mM}$ EGTA, $2 \mathrm{mM}$ pefabloc, $80 \mathrm{mM} \mathrm{NaCl}, 20 \mathrm{mM}$ DTT) by centrifugation for $110 \mathrm{~min}$ at 50000 r.p.m. and $4^{\circ} \mathrm{C}$. Fractions $(500 \mu \mathrm{l})$ were collected by upward displacement with continuous monitoring of the absorbance profile. Each fraction was analysed for protein content following ethanol precipitation, washing to remove all sucrose and resuspension in SDS-sample buffer. DNA was extracted from the fractions using phenol/chloroform and enriched by ethanol precipitation. DNA pellets were washed, resuspended in $\mathrm{H}_{2} \mathrm{O}$ and analysed on a $1 \%$ agarose gel. Subcellular fractionations was carried out using a Subcellular Protein Fractionation kit (Thermo Fisher Scientific) following the supplier's instructions.

To fractionate cells into soluble and insoluble fractions for deubiquitination assays, the cells were washed in ice-cold PBS and lysed on the plate in gentle lysis buffer (PBS+0.5\% Triton X-100, $5 \mathrm{mM}$ EDTA, $20 \mathrm{mM}$ DTT, $0.05 \mathrm{mM}$ Pefabloc) for $20 \mathrm{~min}$ with shaking. The cells were collected and centrifuged at 13000 r.p.m. for $5 \mathrm{~min}$. The pellet was resuspended in deubiquitination buffer ( $50 \mathrm{mM}$ Hepes pH 7.8, $50 \mathrm{mM}$ $\mathrm{NaCl}, 1 \mathrm{mM}$ EDTA, $10 \mathrm{mM}$ DTT and $5 \%$ glycerol) and incubated for $30 \mathrm{~min}$ at $30^{\circ} \mathrm{C}$ in the absence or presence of HAUSP ( $50 \mathrm{ng}$; Boston Biochem).

DNA-binding assays. Electrophoretic mobility-shift assays (EMSAs) were carried out as described previously. ${ }^{27}$ Briefly, indicated amounts of p53 were incubated with $40 \mathrm{ng}$ of ${ }^{33} \mathrm{P}$-labelled oligonucleotides for p21, BAX and MDM2 promoter (see Supplementary Table 1 for details of oligonucleotides), in EMSA buffer (20 mM HEPES (pH 7.5), $50 \mathrm{mM} \mathrm{KCl,} 5 \%$ glycerol, $0.4 \mathrm{mM} \mathrm{DTT}, 0.1 \mathrm{mg} / \mathrm{ml}$ 
BSA, $0.05 \%$ Triton X-100, $0.125 \mathrm{mg} / \mathrm{ml}$ poly(dl-dC) and $0.04 \mathrm{mg} / \mathrm{ml}$ salmon sperm DNA) for $30 \mathrm{~min}$ at RT. The samples were analysed by PAGE $(5 \%$ gel) and radiolabelled bands were detected using a Storm840 phosphoimager (GE Healthcare).

For filter-binding analysis, DNA-binding assays $(25 \mu \mathrm{l})$ contained $10 \mathrm{ng}$ of a $5^{5}-{ }^{33} \mathrm{P}$-end-labelled $66 \mathrm{bp}$ fragment from the p21WAF1 promoter and 10-fold molar excess of unlabelled salmon sperm DNA in buffer containing $25 \mathrm{mM}$ HEPES-KOH (pH 7.8), $5 \mathrm{mM} \mathrm{MgCl}_{2}, 2 \mathrm{mM}$ DTT and $50 \mathrm{mM} \mathrm{KCl}$. Following the addition of p53 (ubiquitinated or non-ubiquitinated), the samples were incubated at $25^{\circ} \mathrm{C}$ for $30 \mathrm{~min}$. The reactions were filtered through nitrocellulose filters (HAWP, $0.22 \mu \mathrm{M}, 13 \mathrm{~mm}$ Millipore), washed with the above buffer $(250 \mu \mathrm{l})$ and equilibrated at the incubation temperature. Radiolabeled DNA retained on the filters was quantified using a Storm840 phosphoimager (GE Healthcare).

For immobilized DNA-binding assays, microtiter plates were coated with streptavidin (1 $\mu \mathrm{g} /$ well in PBS) and incubated with biotin-tagged C1 oligonucleotide $(60 \mathrm{pmol})$ for $1 \mathrm{~h}$. Unbound oligonucleotide was removed by washing and nonreactive sites blocked using $3 \%$ (w/v) BSA in PBS. A titration of IRF-1 (unmodified or ubiquitinated) in $25 \mathrm{mM}$ HEPES, pH 7.5, $50 \mathrm{mM} \mathrm{KCl}, 10 \mathrm{mM} \mathrm{MgCl}, 5 \%$ (v/v) glycerol, $0.1 \%$ (v/v) Tween-20 was added for $1 \mathrm{~h}$. After washing, binding was detected using anti-IRF-1 mAb and electrochemical luminescence quantified using a luminometer (Labsystems, DYNEX Technology, Chantilly, VA, USA).

Generation of models using HADDOCK. Models of IRF-1 and p53 DBD conjugated to ubiquitin were generated using the HADDOCK webserver ${ }^{42,43}$ (as described previously ${ }^{29}$ ). The $\mathrm{C}$ terminus of the p53 DBD crystal structure (PDB:1TUP, resolved at $2.2 \AA^{44}$ ) was extended from residue 291 by grafting residue 292 from $2 \mathrm{AHI}$ (resolved at $1.85 \AA^{45}$ ) onto 1TUP. For the model, the C-terminal glycine residue of ubiquitin $\left(\mathrm{Gly}^{76}\right)$ was selected as the active residue on ubiquitin (PDB:1UBQ, resolved at $1.8 \AA^{40}$ ) and Lys ${ }^{292}$ on p53 or Lys ${ }^{78}$ on IRF-1 (PDB:1IF1, resolved at $3 \AA^{46}$ ), was chosen as the active residue in the p53 or IRF-1 crystal structure, respectively. No passive residues were selected. From the output, the three best structures of the four clusters with the best HADDOCK score were used for analysis (see Supplementary Figure 6). The models were produced in the absence of DNA and subsequently overlaid with the DBD complexed to DNA as seen in the crystal structure. Any complex exhibiting a major clash between the position of ubiquitin and DNA was discarded, and the remaining model was used as the starting structure for a $40 \mathrm{~ns}$ molecular dynamic (MD) simulation in an explicit solvent system. Electrostatic surface analysis of the IRF-1/p53 DBD:monoubiquitin complex were carried out using APBS (Adaptive Poisson-Boltzmann Solver ${ }^{47}$ ) in PyMOL v1.4.1 (http://www.pymol.org).

Molecular dynamic simulations. To model the interactions of DNA with p53 or IRF-1 in its ubiquitinated or unmodified forms, the (extended) crystal structures of the p53 DBD (PDB: 1TUP, 2AHI) or IRF-1 DBD (PDB: 1IF1) and the model of monoubiquitinated p53 or IRF-1 DBD in complex with DNA as generated above were used. The $\mathrm{N}$ - and C-termini of the p53 DBD were capped with acetyl (ACE) and $\mathrm{N}$-methyl (NME), respectively, to keep them neutral. Molecular dynamics simulations were performed with the SANDER module of the AMBER (Assisted Model Building Refinement) 9 package (http://ambermd.org/) together with the ff99SB forcefield. The antechamber and LEaP modules were used to set up the simulation. Systems were solvated in a TIP3P water box with walls at least $8 \AA$ away from any protein atom and net charges on the protein were neutralized using counter ions as required $\left(20-26 \mathrm{Na}^{+}\right)$. To simulate a covalent linkage, a distance restraint between Gly ${ }^{76}$ of ubiquitin and Lys ${ }^{292}$ of $p 53$ or Lys ${ }^{78}$ of IRF-1 (between 1.2 and $2 \AA$ ) was created using a DISANG file in AMBER. A brief energy minimization was carried out followed by heating of the systems to $300 \mathrm{~K}$ and subsequent MD simulations were performed under constant pressure $(1 \mathrm{~atm})$ and temperature $(300 \mathrm{~K})$ using the Sander module. The structures were stored every $2 \mathrm{ps}$ as described before. ${ }^{48}$ The free energies of binding $\left(\Delta G_{\text {bind }}\right)$ of the p53 DBD \pm ubiquitin to DNA were computed and visualizations were carried out as described earlier. ${ }^{48}$ The figures were prepared using PyMOL.

\section{Conflict of Interest}

The authors declare no conflict of interest.

Acknowledgements. VL was funded by a SULSA studentship, MGM by a CRUK Scottish Power alliance studentship, both awarded to KLB. VL obtained a training award from the EACR to work for 3 months with $C V$. BR receives funding from Petplan. NG is an MRC Senior Fellow (MR/J00913X/1). We thank Dr Maria Mativa for preparation of the pcDNA3.1 IRF-1 $\mathrm{K}$ to $\mathrm{R}$ mutants.

1. Lemon B, Tjian R. Orchestrated response: a symphony of transcription factors for gene control. Genes Dev 2000; 14: 2551-2569.

2. Naar AM, Lemon BD, Tjian R. Transcriptional coactivator complexes. Annu Rev Biochem 2001; 70: 475-501.

3. Ee G, Lehming N. How the ubiquitin proteasome system regulates the regulators of transcription. Transcription 2012; 3: 235-239.

4. Geng F, Wenzel S, Tansey WP. Ubiquitin and proteasomes in transcription. Annu Rev Biochem 2012; 81: 177-201.

5. Landre V, Rotblat B, Melino S, Bernassola F, Melino G. Screening for E3-ubiquitin ligase inhibitors: challenges and opportunities. Oncotarget 2014; 5: 7988-8013.

6. Brooks CL, Gu W. p53 regulation by ubiquitin. FEBS Lett 2011; 585: 2803-2809.

7. Hock $A$, Vousden $\mathrm{KH}$. Regulation of the p53 pathway by ubiquitin and related proteins. Int J Biochem Cell Biol 2010; 42: 1618-1621.

8. Soussi T, Wiman KG. TP53: an oncogene in disguise. Cell Death Differ 2016; 22: 1239-1249.

9. Shloush J, Vlassov JE, Engson I, Duan S, Saridakis V, Dhe-Paganon S et al. Structural and functional comparison of the RING domains of two p53 E3 ligases, Mdm2 and Pirh2. J Biol Chem 286: 4796-4808.

10. Chan WM, Mak MC, Fung TK, Lau A, Siu WY, Poon RY. Ubiquitination of p53 at multiple sites in the DNA-binding domain. Mol Cancer Res 2006; 4: 15-25.

11. Maki CG, Howley PM. Ubiquitination of p53 and p21 is differentially affected by ionizing and UV radiation. Mol Cell Biol 1997; 17: 355-363.

12. Wallace M, Worrall E, Pettersson S, Hupp TR, Ball KL. Dual-site regulation of MDM2 E3-ubiquitin ligase activity. Mol Cell 2006; 23: 251-263.

13. Le Cam L, Linares LK, Paul C, Julien E, Lacroix M, Hatchi E et al. E4F1 is an atypical ubiquitin ligase that modulates p53 effector functions independently of degradation. Cell 2006; 127: 775-788.

14. Lai Z, Ferry KV, Diamond MA, Wee KE, Kim YB, Ma J et al. Human mdm2 mediates multiple mono-ubiquitination of p53 by a mechanism requiring enzyme isomerization. $J$ Biol Chem 2001; 276: 31357-31367.

15. Wu H, Leng RP. UBE4B, a ubiquitin chain assembly factor, is required for MDM2-mediated p53 polyubiquitination and degradation. Cell Cycle 2011; 10: 1912-1915.

16. Wang $X$, Wang J, Jiang X. MdmX protein is essential for Mdm2 protein-mediated p53 polyubiquitination. J Biol Chem 2011; 286: 23725-23734.

17. Vassilev LT. Small-molecule antagonists of p53-MDM2 binding: research tools and potential therapeutics. Cell Cycle 2004; 3: 419-421.

18. Wawrzynow B, Pettersson S, Zylicz A, Bramham J, Worrall E, Hupp TR et al. A function for the RING finger domain in the allosteric control of MDM2 conformation and activity. $J$ Biol Chem 2009; 284: 11517-11530.

19. Brooks CL, Gu W. p53 ubiquitination: Mdm2 and beyond. Mol Cell 2006; 21: 307-315.

20. Pamment J, Ramsay E, Kelleher M, Dornan D, Ball KL. Regulation of the IRF-1 tumour modifier during the response to genotoxic stress involves an ATM-dependent signalling pathway. Oncogene 2002; 21: 7776-7785.

21. Kim W, Bennett EJ, Huttlin EL, Guo A, Li J, Possemato A et al. Systematic and quantitative assessment of the ubiquitin-modified proteome. Mol Cell 44: 325-340.

22. Honda R, Tanaka H, Yasuda $H$. Oncoprotein MDM2 is a ubiquitin ligase E3 for tumor suppressor p53. FEBS Lett 1997; 420: 25-27.

23. Brooks CL, Li M, Gu W. Monoubiquitination: the signal for p53 nuclear export? Cell Cycle 2004; 3: 436-438.

24. Carter S, Bischof O, Dejean A, Vousden $\mathrm{KH}$. C-terminal modifications regulate MDM2 dissociation and nuclear export of p53. Nat Cell Biol 2007; 9: 428-435.

25. Gilbert N, Allan J. Distinctive higher-order chromatin structure at mammalian centromeres. Proc Natl Acad Sci USA 2001; 98: 11949-11954.

26. Gilbert N, Thomson I, Boyle S, Allan J, Ramsahoye B, Bickmore WA. DNA methylation affects nuclear organization, histone modifications, and linker histone binding but not chromatin compaction. J Cell Biol 2007; 177: 401-411.

27. Hupp TR, Lane DP. Allosteric activation of latent p53 tetramers. Curr Biol 1994; 4: 865-875.

28. McKinney K, Mattia M, Gottifredi V, Prives $C$. p53 linear diffusion along DNA requires its $C$ terminus. Mol Cell 2004; 16: 413-424.

29. Landre V, Pion E, Narayan V, Xirodimas DP, Ball KL. DNA-binding regulates site-specific ubiquitination of IRF-1. Biochem J 2013; 449: 707-717.

30. Archer CT, Delahodde A, Gonzalez F, Johnston SA, Kodadek T. Activation domaindependent monoubiquitylation of Gal4 protein is essential for promoter binding in vivo. J Biol Chem 2008; 283: 12614-12623.

31. Salghetti SE, Caudy AA, Chenoweth JG, Tansey WP. Regulation of transcriptional activation domain function by ubiquitin. Science 2001; 293: 1651-1653.

32. van der Horst $A$, de Vries-Smits $A M$, Brenkman $A B$, van Triest $M H$, van den Broek $N$, Colland $\mathrm{F}$ et al. FOXO4 transcriptional activity is regulated by monoubiquitination and USP7/ HAUSP. Nat Cell Biol 2006; 8: 1064-1073.

33. Bres V, Kiernan RE, Linares LK, Chable-Bessia C, Plechakova O, Treand C et al. A non-proteolytic role for ubiquitin in Tat-mediated transactivation of the HIV-1 promoter. Nat Cell Biol 2003; 5: 754-761. 
34. Wu RC, Feng Q, Lonard DM, O'Malley BW. SRC-3 coactivator functional lifetime is regulated by a phospho-dependent ubiquitin time clock. Cell 2007; 129: 1125-1140.

35. Wilcox AJ, Laney JD. A ubiquitin-selective AAA-ATPase mediates transcriptional switching by remodelling a repressor-promoter DNA complex. Nat Cell Biol 2009; 11: 1481-1486.

36. Geng F, Wenzel S, Tansey WP. Ubiquitin and proteasomes in transcription. Annu Rev Biochem 2012; 81: 177-201.

37. Lickwar CR, Mueller F, Hanlon SE, McNally JG, Lieb JD. Genome-wide protein-DNA binding dynamics suggest a molecular clutch for transcription factor function. Nature 484: 251-255.

38. Tafvizi A, Huang F, Fersht AR, Mirny LA, van Oijen AM. A single-molecule characterization of p53 search on DNA. Proc Natl Acad Sci USA 108: 563-568.

39. Cary PD, King DS, Crane-Robinson C, Bradbury EM, Rabbani A, Goodwin GH et al. Structural studies on two high-mobility-group proteins from calf thymus, HMG-14 and HMG-20 (ubiquitin), and their interaction with DNA. Eur J Biochem 1980; 112: 577-580.

40. Vijay-Kumar S, Bugg CE, Cook WJ. Structure of ubiquitin refined at $1.8 \mathrm{~A}$ resolution. J Mol Biol 1987; 194: 531-544.

41. Pion E, Narayan V, Eckert M, Ball KL. Role of the IRF-1 enhancer domain in signalling polyubiquitination and degradation. Cell Signal 2009; 21: 1479-1487.

42. de Vries SJ, van Dijk AD, Krzeminski M, van Dijk M, Thureau A, Hsu V et al. HADDOCK versus HADDOCK: new features and performance of HADDOCK2.0 on the CAPRI targets. Proteins 2007; 69: 726-733.
43. Dominguez C, Boelens $R$, Bonvin AM. HADDOCK: a protein-protein docking approach based on biochemical or biophysical information. J Am Chem Soc 2003; 125: 1731-1737.

44. Cho Y, Gorina S, Jeffrey PD, Pavletich NP. Crystal structure of a p53 tumor suppressor-DNA complex: understanding tumorigenic mutations. Science 1994; 265: 346-355.

45. Kitayner M, Rozenberg H, Kessler N, Rabinovich D, Shaulov L, Haran TE et al. Structural basis of DNA recognition by p53 tetramers. Mol Cell 2006; 22: 741-753.

46. Escalante CR, Yie J, Thanos D, Aggarwal AK. Structure of IRF-1 with bound DNA reveals determinants of interferon regulation. Nature 1998; 391: 103-106.

47. Baker NA, Sept D, Joseph S, Holst MJ, McCammon JA. Electrostatics of nanosystems: application to microtubules and the ribosome. Proc Natl Acad Sci USA 2001; 98: 10037-10041.

48. Joseph TL, Madhumalar A, Brown CJ, Lane DP, Verma CS. Differential binding of p53 and nutlin to MDM2 and MDMX: computational studies. Cell Cycle 2010; 9: 1167-1181.

49. Vassilev LT, Vu BT, Graves B, Carvajal D, Podlaski F, Filipovic Z et al. In vivo activation of the p53 pathway by small-molecule antagonists of MDM2. Science 2004; 303: 844-848.

50. Shloush J, Vlassov JE, Engson I, Duan S, Saridakis V, Dhe-Paganon S et al. Structural and functional comparison of the RING domains of two p53 E3 ligases, Mdm2 and Pirh2. J Biol Chem 2011; 286: 4796-4808.

Supplementary Information accompanies this paper on Cell Death and Differentiation website (http://www.nature.com/cdd) 